Pacific Journal of Mathematics

ON A CONSTRAINED VARIATIONAL PROBLEM AND THE
SPACES OF HORIZONTAL PATHS 


\section{ON A CONSTRAINED VARIATIONAL PROBLEM AND THE SPACES OF HORIZONTAL PATHS}

\section{ZHONG GE}

In this paper we study geometry associated to an isoholonomy variational problem on a fat bundle. We prove that the energy function satisfies the Palais-Smale condition on the space of horizontal paths. We blow up the singularity of the horizontal loop space. Then we study the closed geodesics. We relate the number of connected components of the space of loops with trivial holonomy to the topology of the fat bundle.

1. Introduction. In this paper we will study geometry associated to the following constrained variational problem:

Problem 1. Consider a principal $G$-bundle ( $G$ compact) over a compact Riemannian manifold $M$

$$
\pi: F \rightarrow M
$$

with a given connection form $\omega$ on $F$. Fixing two points $x_{0}, x_{1} \in F$, consider the space of $H^{1}$-horizontal paths from $x_{0}$ to $x_{1}$, denoted by $\Omega F\left(x_{0}, x_{1}\right)$. Then the problem is to find a horizontal path $\gamma_{0} \in$ $\Omega F\left(x_{0}, x_{1}\right)$ such that

$$
E\left(\gamma_{0}\right)=\min _{\gamma \in \Omega F\left(x_{0}, x_{1}\right)} E(\gamma),
$$

where $E(\gamma)=\int_{0}^{1}\left\|\pi_{*} \gamma(t)\right\|^{2} d t$. Recall that a path $\gamma$ in $F$ is horizontal if

$$
\omega(\dot{\gamma})=0 .
$$

The most important case in application (cf. [18]) is when the two end points lie on the same fiber, and the problem reduces to the following form:

Problem 2. Let $\Omega M\left(x_{0}, H_{0}\right)$ be the space of $H^{1}$-loops on $M$ based at $x_{0} \in M$ with holonomy $H_{0}$, then the problem is to find a loop $\gamma_{0}$ such that

$$
E\left(\gamma_{0}\right)=\min _{\gamma \in \Omega M\left(x_{0}, H_{0}\right)} E(\gamma),
$$

where $E(\gamma)=\int_{0}^{1}\|\dot{\gamma}\|^{2} d t$ 
Define a metric on $F$ by

$$
d(x, y)=\min _{\gamma \in \Omega F(x, y)}(E(\gamma))^{1 / 2},
$$

which is called the Carnot-Carathéodory metric on $F$ (see the discussion in Gromov [10], Koranyi [15]). This metric has appeared in various contexts: the collapse of Riemannian metrics (cf. [10], [15], [17], [18], [19]), the growth of volumes in Riemannian manifold (cf. [19]), pseudo-hermitian structure on strongly pseudo-convex CR manifold (cf. [15], [24]), Vakanomic mechanics (cf. [1]), control theory (cf. [6]), microbiology and quantum physics (cf. [18]). This metric is also the underlying geometry of hypoelliptic operators (cf. [4], [22]).

We will study this problem from the point of view of sub-Riemannian geometry. It is well known that the path space plays a very important role in Riemannian geometry (cf. [10], [14]), and in the case of sub-Riemannian geometry, we expect that the horizontal path space will play a similar role.

In this paper we study the simplest case: $F$ is a fat bundle in the sense of Weinstein, so that the Carnot-Carathéodory metric is strongly bracket generating (cf. Strichartz [20]). In this case $\Omega F(x, y)$ is smooth if $x \neq y$, and $\Omega F(x, x)$ has a singularity at the constant loop. One of our main results is the resolution of this singularity and its application to the study of closed geodesics.

We first develop some analytical tools. In the second section we study the holonomy map and show that the energy function $E$ on $\Omega M\left(x_{0}, H_{0}\right)$ satisfies the P-S condition. In particular, we show that geodesics are smooth, which is a special case of regularity results for geodesics by Bar, Hamenstadt, Taylor, see [11], [22], [29] for details. In their treatment they only require that the horizontal bundle satisfies Hörmander's condition. In particular, their work implies the equivalence of the Lagrangian approach by Carathéodory, Hermann (cf. [13]) et al. and the Hamiltonian approach by Rayner [28] et al.

In the third section we define the concept of conjugate points and compute the Morse index of the energy $E$ on $\Omega F\left(x_{0}, x_{1}\right)$ at a geodesic in terms of conjugate points as in Riemannian geometry. This amounts to the study of a 2nd-order differential-integral operator (the Jacobi field) subject to a constraint on the line. Although our result takes the same form as the Morse index theorem, its proof is more complicated than that of the Riemannian case. Then we show that there are arbitrary nearby conjugate points, thus sharpen a result of Rayner [28] and Strichartz [20]. 
In the fourth section we study the horizontal loop space, both free and based. We describe a blowing up of the singularity and show that there is a canonical correspondence between the blow-up and certain two-step nilpotent Lie algebras. For the free horizontal loop space, we show that there is a nature smooth $S^{1}$ action on its blow-up.

In the fifth section we apply the results obtained in $\S 4$ to the closed geodesics problem. Since the horizontal loop space is singular at the constant loops, we will consider either the punctured horizontal loop space, or the blow-up of the horizontal loop space. In particular, we show that the number of connected components on the based horizontal loop space is that of the based loop space on $F$. This somehow generalizes a result of Smale [27]. Further results concerning the topology of horizontal loop space will appear elsewhere.

In the appendix we derive the Jacobi field for the variational problem on $M$. However, we have not been able to define the curvature (cf. Strichartz [20] for a discussion).

We wish to thank Richard Montgomery and Alan Weinstein, to whom much of this paper owes its existence (in particular, Montgomery carefully read the manuscript and pointed out a number of mistakes) and Jerry Marsden, Albert Sheu for their help. Also we hope to thank the referee for suggesting improvement in the organization and English of this paper.

\section{Horizontal paths spaces.}

2.1. Spaces of horizontal paths and Hörmander's condition. Let $H$ be the horizontal subbundle in TF . A classical result of Chow says that if the connection satisfies Hörmander's condition and $F$ is connected, then for every $x_{1}, x_{2} \in F$, there is a horizontal $H^{1}$ path connecting them (cf. [6], [20]).

The following result is due to Weinstein [26]. For the sake of completeness we include the proof.

Proposition 2.1. If $F$ is connected and the connection satisfies Hörmander's condition, then the spaces $\Omega F\left(x_{1}, x_{2}\right)$, consisting of horizontal $H^{1}$ paths from $x_{1}$ to $x_{2}$, are all homotopic to each other.

Proof. We will only prove that $\Omega F\left(x_{0}, x_{0}\right)$ is homotopic to $\Omega F\left(x_{0}, x_{1}\right)$. Take a horizontal path $\gamma_{0}$ starting at $x_{0}$ and ending at $x_{1}$. Define a map $A: \Omega F\left(x_{0}, x_{0}\right) \rightarrow \Omega F\left(x_{0}, x_{1}\right)$ by 
and a map $B: \Omega F\left(x_{0}, x_{1}\right) \rightarrow \Omega F\left(x_{0}, x_{0}\right)$ by

$$
\gamma \rightarrow \gamma_{0}^{-1} \gamma
$$

where $\gamma_{0} \gamma$ denotes the path

$$
\gamma_{0} \gamma(t)= \begin{cases}\gamma(2 t), & 0 \leq t \leq 1 / 2 \\ \gamma_{0}(2 t-1), & 1 / 2 \leq t \leq 1\end{cases}
$$

and $\gamma^{-1}(t)$ denotes the path

$$
\gamma^{-1}(t)=\gamma(1-t)
$$

Now $A B: \Omega F\left(x_{0}, x_{1}\right) \rightarrow \Omega F\left(x_{0}, x_{1}\right), \gamma \rightarrow \gamma_{0} \gamma_{0}^{-1} \gamma$ is homotopic to the identity. In fact, let $\gamma_{\varepsilon}$ be the paths

$$
\gamma_{\varepsilon}(t)=\gamma_{0}(\varepsilon(1-t))
$$

then the map $L_{\varepsilon}$

$$
\gamma \rightarrow \gamma_{\varepsilon} \gamma_{\varepsilon}^{-1} \gamma
$$

is the identity map when $\varepsilon=0$, and $L_{1}=A B$. Similarly $B A$ is also homotopic to the identity map.

2.2. Linear O.D.E. 's on Lie Groups. In this subsection we will study linear O.D.E.'s on a Lie group, which will be used in the study of the holonomy map.

Consider a (time-dependent) linear O.D.E. on Lie group $G$,

$$
\dot{q}(t)=T L_{q(t)} B(t)
$$

where $B$ is a $g$-valued function. If $B$ is continuous with respect to $t$, then the solution $q(t)$ can be written as (cf. [8])

$$
q(t)=\lim _{N \rightarrow \infty} \exp \left(t B\left(t_{0}\right) / N\right) \cdots \exp \left(t B\left(t_{N}\right) / N\right)
$$

where $0=t_{0}<t_{1}<\cdots<t_{N}=t$. If $B(t)$ is not continuous but only integrable, then (2.2) is no longer valid. Nevertheless, (2.2) is valid in the approximate sense, as we will see below. First we specify the space of $B$.

Definition 2.1. $L([0, T], g)$ is the space of time-dependent vector fields $B(t)$ such that

$$
\|B\|=\int_{0}^{T}\|B(t)\| d t<\infty .
$$

We smooth the non-smooth $B(t)$ in the usual way. Take a smooth cutoff function $\rho(t)$ which vanishes outside $-1<t<1$, and has total integral 1 in $[-1,1]$. We define

$$
B_{h}(t)=h^{-1} \int_{-\infty}^{\infty} B(s) \rho((t-s) / h) d s .
$$


Proposition 2.2. Let $q_{h}(t)$ be the solution of the time-dependent O.D.E. on $G$

$$
\dot{q}_{h}(t)=\left(L_{q_{h}(t)}\right)_{*} B_{h}(t) .
$$

Then as $h \rightarrow 0, q_{h}(t)$ converges to $q(t)$, the solution to

$$
\dot{q}=\left(L_{q}\right)_{*} B(t)
$$

Proof. Let the Lie algebra $g$ be a subalgebra in the Lie algebra of $k \times k$ matrices $\operatorname{gl}(k)$ for suitable $k$; then (2.4) ((2.3) respectively) can be extended to be a O.D.E. on matrices,

$$
\dot{A}(t)=A(t) B(t) \quad\left(\dot{A}_{h}(t)=A_{h}(t) B_{h}(t) \text { respectively }\right) .
$$

The solution can be written as

$$
A(t)=1+\sum_{k=1}^{\infty} \int \cdots \int_{0 \leq t_{1} \leq \cdots \leq t_{k} \leq t} B\left(t_{1}\right) \cdots B\left(t_{k}\right) d t_{1} \cdots d t_{k} / k ! .
$$

It is easy to prove that

$$
\begin{gathered}
\left\|\int \cdots \int_{0 \leq t_{1} \leq \cdots \leq t_{k} \leq t}\left(B\left(t_{1}\right) \cdots B\left(t_{k}\right)-B_{h}\left(t_{1}\right) \cdots B_{h}\left(t_{k}\right)\right) d t_{1} \cdots d t_{k}\right\| \\
\leq k\left(\int_{0}^{t}\left(\|B(t)\|+\left\|B_{h}(t)\right\|\right) d t\right)^{k-1} \cdot \int_{0}^{t}\left\|B_{h}(t)-B(t)\right\| d t
\end{gathered}
$$

so the proposition follows.

2.3. The holonomy map. We will study the holonomy map which assigns a loop to its holonomy

$$
H: \Omega M\left(x_{0}\right) \rightarrow G
$$

where $\Omega M\left(x_{0}\right)$ is the space of $H^{1}$-loops on $M$ based at $x_{0}$. We first prove a bound on the holonomy of a loop in terms of its length ("isoperimetric inequality"). Let $D\left(q_{1}, q_{2}\right)$ denote the distance function of the bi-invariant metric \|\| on $G$, and \|\|$_{F}$ the Kaluza-Klein metric on $F$ (cf. [18]).

Proposition 2.3. Let $\gamma$ be a loop on $M$. Then

$$
D(H(\gamma), e) \leq\|\omega\| \text { length }(\gamma)
$$

where

$$
\|\omega\|=\max _{x \in F} \max _{y \in T_{x} F} \frac{\|\omega(y)\|}{\|y\|_{F}}
$$


Proof. Take local coordinates $(x, q)$ on $F$. Then $\omega$ can be written as

$$
\omega(x, q)=\omega_{0}(q)+\bar{q}^{1}(t) A(x(t)) q(t) x(t) .
$$

We may confine ourself to the case where $\gamma$ lies wholly in a single chart $U_{\alpha}$ on $M$ (for which we have of course $\pi^{-1}\left(U_{\alpha}\right)=G \times U_{\alpha}$ ). Suppose that the loop is $t \rightarrow x(t)$ and the horizontal path is given by $t \rightarrow(x(t), q(t))$. Let $B(t)=\dot{x}^{\mu}(t) A_{\mu}(x(t))$; then $q(t)$ is the solution of the O.D.E.

$$
\dot{q}-\left(L_{B(t)}\right)_{*} q=0 .
$$

As is shown in $\S 2.2$, we may only work with smooth $B(t)$. By the triangle inequality and the bi-invariance of the metric $D$, we see that

$$
\begin{aligned}
D\left(q_{B}(t), e\right) & =\lim _{N \rightarrow \infty} D\left(\exp \left(t B\left(t_{0}\right) / N\right) \cdots \exp \left(t B\left(t_{N}\right) / N\right), e\right) \\
& \leq \lim _{N \rightarrow \infty} \sum_{i=1}^{N} D\left(\exp \left(t B\left(t_{i}\right) / N\right), e\right)=\lim _{N \rightarrow \infty} \sum_{i=1}^{N}\left\|t B\left(t_{i}\right)\right\| / N \\
& \leq \int_{0}^{t}\|B(t)\| d t .
\end{aligned}
$$

So

$$
\begin{aligned}
D\left(q_{B}(t), e\right) & \leq \int_{0}^{t}\|B(t)\| d t \leq \int_{0}^{t}\left\|\dot{x}^{\mu}(t) A(x(t))\right\| d t \\
& \leq\|A\| \int_{0}^{t}\|\dot{x}(t)\| d t
\end{aligned}
$$

where

$$
\|A\|=\max _{x}(\|A(x(t))\| /\|x\|) \leq\|\omega\|
$$

Now we prove the continuity of the holonomy map under some extra assumption on the energy function $E$.

Proposition 2.4. Suppose that $M \rightarrow R^{k}$ is an isometrical embedding. If $\gamma_{n} \in \Omega M\left(x_{0}\right)$ is a sequence on which $E$ is bounded, $E\left(\gamma_{n}\right)<M_{0}<\infty$, and

$$
\lim _{n \rightarrow \infty} \int_{0}^{t}\left(\left\|\dot{\gamma}_{n}(t)-\dot{\gamma}(t)\right\|+\left\|\gamma_{1}(t)-\gamma_{2}(t)\right\|\right) d t=0,
$$

then

$$
\lim _{n \rightarrow \infty} H\left(\gamma_{n}\right)=H(\gamma) .
$$


Proof. Suppose the horizontal path covering $\gamma_{1} \quad\left(\gamma_{2}\right.$ respectively) is $\left(x_{1}(t), q_{B_{1}}(t)\right)\left(\left(x_{2}(t), q_{B_{2}}(t)\right)\right.$ respectively. We need only to prove that

$$
D\left(q_{B_{1}}(t), q_{B_{2}}(t)\right) \leq M \int_{0}^{t}\left\|B_{1}(t)-B_{2}(t)\right\| d t
$$

where $B_{i}(t)=\dot{x}_{i}^{\mu}(t) A(x(t)), i=1,2$.

W.o.l.g. we need only to prove $(2.6)$ for smooth $B_{1}, B_{2}$.

$$
\begin{gathered}
D\left(q_{B_{1}}(t), q_{B_{2}}(t)\right)=\lim _{N \rightarrow \infty} D\left(\exp \left(t B_{1}\left(t_{1}\right) / N\right) \cdots \exp \left(t B_{1}\left(t_{N}\right) / N\right),\right. \\
\left.\exp \left(t B_{2}\left(t_{1}\right) / N\right) \cdots \exp \left(t B_{2}\left(t_{N}\right) / N\right)\right) \\
\leq \lim _{N \rightarrow \infty} \sum_{i=1}^{N} D\left(\exp \left(t B_{1}\left(t_{1}\right) / N\right) \cdots \exp \left(t B_{1}\left(t_{i-1}\right) / N\right) \exp \left(t B_{2}\left(t_{i}\right) / N\right)\right. \\
\cdots \exp \left(t B_{2}\left(t_{1}\right) / N\right), \exp \left(t B_{1}\left(t_{1}\right) / N\right) \\
\cdots \exp \left(t B_{1}\left(t_{i}\right) / N\right) \exp \left(t B_{2}\left(t_{i+1}\right) / N\right) \\
\left.\cdots \exp \left(t B_{2}\left(t_{N}\right) / N\right)\right) \\
=\lim _{N \rightarrow \infty} \sum_{i=1}^{N} D\left(\exp \left(t B_{1}\left(t_{i}\right) / N\right), \exp \left(t B_{2}\left(t_{i}\right) / N\right)\right) \\
\leq C \lim _{N \rightarrow \infty} \sum_{i=1}^{N}\left\|t B_{1}\left(t_{i}\right)-t B_{2}\left(t_{i}\right)\right\| / N \\
\leq M \int_{0}^{t}\left\|B_{1}(t)-B_{2}(t)\right\| d t .
\end{gathered}
$$

So

$$
\begin{aligned}
& D\left(q_{B_{1}}(t), q_{B_{2}}(t)\right) \leq M \int_{0}^{t}\left\|B_{1}(t)-B_{2}(t)\right\| d t \\
& \leq M\left(\int_{0}^{t}\left\|\left(\dot{x}_{1}-\dot{x}_{2}\right) A\left(x_{1}(t)\right)\right\| d t+\int_{0}^{t}\left\|x_{1}\left(A\left(x_{1}\right)\right)-A\left(x_{2}\right)\right\| d t\right) \\
& \leq M\left(\max \|A(x(t))\| \int_{0}^{t}\left\|\dot{x}_{1}-\dot{x}_{2}\right\| d t\right. \\
& \left.\left.\quad+\max \| A_{x}(t)\right)\left\|\int_{0}^{t}\right\| x_{1}-x_{2}\left\|d t \max _{i} \max \right\| x_{i}(t) \|\right) .
\end{aligned}
$$

Since $E(\gamma)<M_{0}<\infty, \max \|x(t)\|$ is universally bounded. Hence

$$
D\left(q_{B_{1}}(t), q_{B_{2}}(t)\right) \leq M^{\prime} \int_{0}^{t}\left(\left\|\dot{x}_{1}(t)-\dot{x}_{2}(t)\right\|+\left\|x_{1}(t)-x_{2}(t)\right\|\right) d t .
$$


In order to obtain the derivative of the holonomy map, we have to study the derivative of the solution to the following O.D.E.

$$
\dot{q}(t)=q(t) B(t)
$$

with respect to $B$. Here $q$ and $b$ are $k \times k$ matrices. We denote its solution by $q_{B(t)}$.

Proposition 2.5.

$$
q_{B+\delta B}(t)-q_{B}(t)-\int_{0}^{t} q_{B}(s) \delta B(s) q_{B}^{-1}(s) d s q_{B}(t)=o(\|\delta B\|) .
$$

Proof. As is shown in $\S 2.2$, we need only to prove (2.7) for smooth $B(t)$. Now

$$
\begin{gathered}
q_{B+\delta B}(t)-q_{B}(t)-\int_{0}^{t} q_{B}(s) \delta B(s) q_{B}^{-1}(s) d s q_{B}(t) \\
=\lim _{N \rightarrow \infty}\left(\exp (t B+\delta B)\left(t_{0}\right) / N\right) \cdots\left(\exp \left(t(B+\delta B)\left(t_{N}\right) / N\right)\right. \\
\left.\quad-\exp \left(t B\left(t_{0}\right) / N\right) \cdots \exp \left(t B\left(t_{N}\right) / N\right)\right) \\
-\int_{0}^{t} q_{B}(s) \delta B(s) q_{B}^{-1}(s) d s q_{B}(t) \\
=\lim _{N \rightarrow \infty} \sum_{i=1}^{N} \exp \left(t(B+\delta B)\left(t_{0}\right) / N\right) \\
\quad \cdots \exp \left(t(B+\delta B)\left(t_{i-1}\right) / N\right) \\
\left.\quad \cdot \exp \left(t(B+\delta B)\left(t_{i}\right) / N\right)-\exp \left(t B\left(t_{i}\right) / N\right)\left(1+\left(\delta B\left(t_{i}\right) / N\right)\right)\right\} \\
\cdots \exp \left(t B\left(t_{i+1}\right) / N\right) \cdots \exp \left(t B\left(t_{N}\right) / N\right) .
\end{gathered}
$$

Now we estimate

$$
\begin{aligned}
& \exp (\left.t\left(B\left(t_{i}\right)+\delta B\left(t_{i}\right)\right) / N\right)-\exp \left(t\left(B\left(t_{i}\right) / N\right)\left(I+\left(\delta B\left(t_{i}\right) t / N\right)\right)\right) \\
&= \exp \left(t B\left(t_{i}\right) / N\right) \alpha\left(\operatorname{ad}\left(t B\left(t_{i}\right) / N\right)\right)\left(t B\left(t_{i}\right) / N\right)+o\left(t\left|\delta B\left(t_{i}\right)\right| / N\right) \\
&= \exp \left(t B\left(t_{i}\right) / N\right) \alpha\left(\operatorname{ad}\left(t B\left(t_{i}\right) / N\right)\left(t \delta B\left(t_{i}\right) / N\right)\right)+o\left(t \mid\left(\delta B\left(t_{i}\right) \mid / N\right)\right) \\
& \quad \text { where } \alpha(t)=1+(x / 2)+\left(x^{2} / 3 !\right)+\cdots=(\exp (x)-1) / x
\end{aligned}
$$

So

$$
q_{B+\delta B}(t)-q_{B}(t)-\int_{0}^{t} q_{B}(s) \delta B(s) q_{B}^{-1}(s) d s q_{B}(t)=o(\|\delta B\|) .
$$

Corollary 2.1. The map $H: \Omega M\left(x_{0}\right) \rightarrow G$ is $C^{1}$ and its derivative has the form

$$
d H\left(\gamma_{\varepsilon}\right) /\left.d \varepsilon\right|_{\varepsilon=0}=-\oint U_{\tau} F_{\omega}(\dot{\gamma}, \delta \gamma) U_{\tau}^{-1} d \tau H(\gamma)
$$


where $U_{\tau}$ denotes the operation of parallel translation along $\gamma$ to the fiber over $\gamma(\tau), F_{\omega}$ is the curvature of $\omega$. Here we loosely use the notation $\operatorname{Ad}_{U} F(\dot{\gamma}, \delta \gamma)=U F(\dot{\gamma}, \delta \gamma) U^{-1}, \delta \gamma=\partial \gamma_{\varepsilon} /\left.\partial \varepsilon\right|_{\varepsilon=0}$.

Proof. We work in local coordinates. Suppose $\gamma$ is given by $t \rightarrow$ $x(t)$ and the horizontal path covering $\gamma$ is given by $t \rightarrow(x(t), q(t))$. Then $q(t)$ is the solution of

$$
\dot{q}(t)=q(t) B(t) ;
$$

here $B(t)=\dot{x}(t) A(x(t))$. So

$$
\begin{aligned}
\delta B(t) & =(\dot{x}+\delta \dot{x})(t) A(x(t)+\delta x(t))-\dot{x}(t) A(x(t)) \\
& =(\dot{x}+\delta \dot{x})(t)\left(A(x(t))+A_{x}(x(t)) \delta x(t)+o(|\delta x|)\right)-\dot{x}(t) A(x(t)) \\
& =\dot{x}(t) A_{x}(x(t)) \delta x(t)+\delta \dot{x}(t) A(x(t))+o\left(|\delta x|^{2}+|\delta \dot{x}|^{2}\right) .
\end{aligned}
$$

Use Proposition 2.5,

$$
\begin{gathered}
\int_{0}^{t} q(s) \delta B(s) q^{-1}(s) d s \\
=\int_{0}^{t} q(s)\left(\dot{x}(s) A_{x}(x(s)) \delta x(s)+\delta \dot{x}(s) A(x(s))\right. \\
\left.\quad+o\left(\left|\delta x^{2}\right|+\left|\delta \dot{x}^{2}\right|\right)\right) q^{-1}(s) d s \\
=\int_{0}^{t}\left\{q(s) \dot{x}(s) A_{x}(x(s)) \delta x(s) q^{-1}(s)\right. \\
\left.\quad+q(s) \delta \dot{x}(s) A(x(s)) q^{-1}(s)\right\} d s+o\left(\|\delta x\|^{2}\right) \\
=\int_{0}^{t}\left\{q(s) \dot{x}(s) A_{x}(x(s)) \delta x(s) q^{-1}(s)\right. \\
-q(s) \dot{x}(s) A(x(s)) \delta x(s) A(x(s)) q(s) \\
-q(s) \delta x(s) A_{x}(x(s)) \dot{x}(s) q^{-1}(s) \\
\left.+q(s) \delta x(s) A(x(s)) \dot{x}(s) A(x(s)) q^{-1}(s)\right\} d s \\
+q(t) \delta x(t) A(x(t)) q^{-1}(t)+o\left(\|\delta x\|^{2}\right) \\
=\int_{0}^{t} q(s) F_{\omega}(\dot{x}(s), \delta x(s)) q^{-1}(s) d s \\
+q(t) \delta x(t) A(x(t)) q^{-1}(t)+o\left(\|\delta x\|^{2}\right) .
\end{gathered}
$$

Now $\delta x(0)=\delta x(1)=0$; hence the corollary follows.

REMARK. Since we have used the left-handed fiber bundle, the above formula is different from that of Montgomery [18].

Now we recall the definition of fat bundles (cf. Weinstein [25]): we say a bundle $F \rightarrow M$ with connection $\omega$ is fat if for every $a \in g^{*}$, 
$a \neq 0$, and horizontal tangent vector $x \in T_{z} F, x \neq 0$, there is a $y \in T_{z} F$, such that

$$
\left\langle a, F_{\omega}(x, y)\right\rangle \neq 0
$$

where $F_{\omega}$ is the curvature of $\omega$.

This definition is equivalent to that (cf. [4]): the set

$$
\Phi=\left\{(x, p) \in T^{*} F,\langle p, H\rangle=0\right\}
$$

forms a symplectic submanifold in $T^{*} F$, where $H$ is the horizontal subbundle of $T F$.

See Bergery [3] for a classification of homogeneous fat bundles with reductive structure group.

COROLLARY 2.2. If the fiber bundle is fat (so that the horizontal bundle is strongly bracket generating), then the space of loops having the same holonomy $H_{0} \neq e, \Omega M\left(x_{0}, H_{0}\right)$, is a smooth Hilbertsubmanifold of $\Omega M\left(x_{0}\right)$. Also, if $H_{0}=e$, then $\Omega M\left(x_{0}, e\right)$ is a smooth submanifold except at the constant loop.

Proof. If the bundle is fat, then the map $H: \Omega M\left(x_{0}\right) \rightarrow g$ is submersive except at the constant loop, so the corollary follows.

2.3. Existence and regularity. In this subsection we will prove the existence of minimizers and smoothness of extremals.

Proposition 2.6. If $\Omega M\left(x_{0}, H_{0}\right)$ is not empty, then the minimizing elements of the energy functional $E$ in $\Omega M\left(x_{0}, H_{0}\right)$ exist. If the fiber function is fat, then the extremals (including the minimizing elements) are smooth.

Proof. Let $\gamma_{n}$ be a minimizing sequence,

$$
\lim _{n \rightarrow \infty} E\left(\gamma_{n}\right)=\inf E(\gamma) \text {. }
$$

By the weak compactness of the unit ball in a Hilbert space, there is a $\gamma_{0} \in \Omega M\left(x_{0}\right)$ and a subsequence of $\left\{\gamma_{n}\right\}$ (which we still denote by $\left.\left\{\gamma_{n}\right\}\right)$ such that

$$
\begin{gathered}
\lim _{n \rightarrow \infty} \max d\left(\gamma_{n}(t), \gamma_{0}(t)\right)=0, \\
\lim _{n \rightarrow \infty} E\left(\gamma_{n}\right) \geq E\left(\gamma_{0}\right),
\end{gathered}
$$

where $d$ is the Carnot-Carathéodory metric on $F$, see the introduction. We only need to prove that $H\left(\gamma_{0}\right)=H_{0}$. Without loss of generality we work in local coordinates. Let $\gamma_{n}$ be $t \rightarrow\left(x_{n}(t), q_{n}(t)\right)$, 
$\gamma$ be $t \rightarrow\left(x_{0}(t), q_{0}(t)\right)$; there is a subsequence (which we still denote by $\gamma_{n}$ ) such that

$$
\int_{0}^{t}\left(\left\|\dot{x}_{n}(t)-\dot{x}_{0}(t)\right\|+\left\|x_{n}(t)-x_{0}(t)\right\|\right) d t \rightarrow 0 \quad \text { as } n \rightarrow \infty .
$$

By Proposition 2.4, $q(t)=\lim _{n \rightarrow \infty} q_{n}(t)$. Hence $H\left(\gamma_{0}\right)=H_{0}$. So $\gamma_{0}$ is a minimizing element.

For the proof of regularity, we just remark that it is a standard result in O.D.E. (cf. [14]).

2.4. The Palais-Smale Condition. Let $E_{0}$ denote the restriction of $E$ to $\Omega M\left(x_{0}, H_{0}\right)$, and $\left\langle\langle,\rangle_{0}\right.$ the metric on $\Omega M\left(x_{0}, H_{0}\right)$ induced from the metric $\left\langle\langle\right.$,$\rangle of \Omega M\left(x_{0}\right)$. using the metric $\langle\langle,\rangle\rangle_{0}$ on $\Omega M\left(x_{0}, H_{0}\right)$ we define the corresponding vector field $\operatorname{grad} E_{0}$. To establish Palais-Smale condition we must show that whenever $\left\{\gamma_{n}\right\}$ is a sequence on which $E$ is bounded and for which $\left\|\operatorname{grad}\left(e_{0}\right) \gamma_{n}\right\|_{0} \rightarrow 0$ when $n \rightarrow \infty,\left\{\gamma_{n}\right\}$ has a convergent subsequence. In this subsection we always assume that $H_{0} \neq e$.

We will also use another topology on $\Omega M\left(x_{0}\right)$ : the $d_{\infty}$-topology on $\Omega M\left(x_{0}\right)$ is defined by the metric

$$
\rho\left(\gamma_{1}, \gamma_{2}\right)=\max _{t \in[0,1]} D\left(\gamma_{1}(t), \gamma_{2}(t)\right)
$$

where $D$ is the distance on $M$.

Let us first examine what the above assumption on $\gamma_{n}$ implies.

Proposition 2.7. Let $\left\{\gamma_{n}\right\} \subset \Omega M\left(x_{0}\right)$ be a sequence in $\Omega M\left(x_{0}\right)$ on which $E$ is bounded. Then $\left\{\gamma_{n}\right\}$ has a subsequence converging in $d_{\infty}$ to a continuous path $\gamma$ on $M$.

For a proof see [14].

The following three propositions will reduce the condition $\left\|\operatorname{grad} E_{0}\left(\gamma_{n}\right)\right\| \rightarrow 0$ to $\left\|\operatorname{grad} E\left(\gamma_{n}\right)+\left(a, \operatorname{grad} H\left(\gamma_{n}\right)\right)\right\| \rightarrow 0$ for some $a \in g^{*}$ (a refined method of Lagrangian multipliers).

Proposition 2.8. If $\left\{\gamma_{n}\right\}$ is a sequence for which

$$
\left\|\operatorname{grad} E_{0}(\gamma)\right\|_{0} \rightarrow 0 \text { as } n \rightarrow \infty,
$$

then there exist $a_{n} \in g^{*}$ such that

$$
\left\|\operatorname{grad} E\left(\gamma_{n}\right)+\left(\operatorname{grad} H\left(\gamma_{n}\right), a_{n}\right)\right\| \rightarrow 0 \text { as } n \rightarrow \infty \text {. }
$$


Proof. Let $\Sigma_{\gamma}$ be the space of $H^{1}$-vector fields $X$ along $\gamma$ such that

$$
(d H(\gamma), X)=0
$$

Then we have

$$
\lim _{n \rightarrow \infty}\left(d E\left(\gamma_{n}\right), X\right)=0,
$$

for $X \in \Sigma_{\gamma_{n}},\|X\| \leq 1$. Let $P_{\gamma}: T_{\gamma} \Omega M\left(x_{0}\right) \rightarrow \Sigma_{\gamma}$ be the orthogonal projection; then from (2.8) we have

$$
\lim _{n \rightarrow \infty}\left(P_{\gamma_{n}} d E\left(\gamma_{n}\right), X\right)=\lim _{n \rightarrow \infty}\left(d E\left(\gamma_{n}\right), P_{\gamma_{n}} X\right)=0
$$

for $\|X\| \leq 1$. But $P_{\gamma} d E(\gamma)=d E(\gamma)-\left(a_{\gamma}, d H(\gamma)\right)$ for some $a_{\gamma} \in g^{*}$, so the proposition is proved.

Proposition 2.9. Suppose that $F \rightarrow M$ is a fat bundle. If $\{\gamma\}$ is a sequence for which $\left\|\operatorname{grad} E\left(\gamma_{n}\right)\right\|_{0} \rightarrow 0$ as $n \rightarrow \infty$, then $\left\{\gamma_{n}\right\}$ has a subsequence (which we still denote by $\left\{\gamma_{n}\right\}$ ) and some $a \in g^{*}$ such that

$$
\left\|\operatorname{grad} E\left(\gamma_{n}\right)+\left(\operatorname{grad} H\left(\gamma_{n}\right), a\right)\right\| \rightarrow 0 \text { as } n \rightarrow \infty .
$$

Proof. Let $\left\{a_{n}\right\}$ be as in Proposition 2.8. We first want to prove that $\left\{a_{n}\right\}$ is a bounded set in $g^{*}$. Assuming the contrary: $a_{n} \rightarrow \infty$, as $n \rightarrow \infty$, then

$$
\left\|\left(\operatorname{grad} E\left(\gamma_{n}\right) /\left\|a_{n}\right\|\right)+\left(\operatorname{grad} H\left(\gamma_{n}\right), \quad\left(a_{n} /\left\|a_{n}\right\|\right)\right)\right\| \rightarrow 0 \text { as } n \rightarrow \infty .
$$

Note that since $M$ is compact, $\|\operatorname{grad} E(\gamma)\|$ is bounded by $E(\gamma)$,

$$
\|\operatorname{grad} E(\gamma)\| \leq M E(\gamma) \text {, }
$$

from which we have

$$
\left\|\left(\operatorname{grad} H\left(\gamma_{n}\right), a_{n} /\left\|a_{n}\right\|\right)\right\| \rightarrow 0 \text { as } n \rightarrow \infty .
$$

Now by Proposition 2.7 we can assume that $a_{n} /\left\|a_{n}\right\| \rightarrow a \neq 0$, and that $\gamma_{n} \rightarrow \gamma$, as $n \rightarrow \infty$, in the $d_{\infty}$-topology, to obtain

$$
(\operatorname{grad} H(\gamma), a)=0,
$$

which is in contradiction with the assumption that the bundle is fat. So $\left\{a_{n}\right\}$ is bounded. W.1.o.g. we assume that $a_{n} \rightarrow a$, as $n \rightarrow \infty$. Then from $\|\operatorname{grad} H(\gamma)\| \leq M E(\gamma)$ we get

$$
\begin{gathered}
\left\|\operatorname{grad} E\left(\gamma_{n}\right)+\left(\operatorname{grad} H\left(\gamma_{n}\right), a_{n}\right)-\left(\operatorname{grad} E\left(\gamma_{n}\right)+\left(\operatorname{grad} H_{a}\left(\gamma_{n}\right), a\right)\right)\right\| \\
=\left\|\left(\operatorname{grad} H\left(\gamma_{n}\right), a_{n}-a\right)\right\| \rightarrow 0 \text { as } n \rightarrow \infty .
\end{gathered}
$$

So the proof is completed. 
By a nature chart around $\gamma \in \Omega M\left(x_{0}\right)$ we understand a chart defined as follows

$$
\exp _{\gamma}: T_{\gamma} \Omega M\left(x_{0}\right) \rightarrow \Omega M\left(x_{0}\right)
$$

is given by

$$
\exp _{\gamma}(X)(t)=\exp _{t}(X(t))
$$

for all $X \in T_{\gamma} \Omega M\left(x_{0}\right)$ and for all $t \in R$. Here $\exp _{t}: T_{\gamma(t)} M \rightarrow$ $M$ is the Riemannian exponential map. $\exp _{\gamma}$ is a diffeomorphism of a neighborhood around the zero-section 0 in $T_{\gamma} \Omega M\left(x_{0}\right)$ onto a neighborhood around $\gamma \in \Omega M\left(x_{0}\right)$, i.e. it is a chart on $\Omega M\left(x_{0}\right)$.

THEOREM 2.1. If $F \rightarrow M$ is a fat bundle and $H_{0} \neq e$, then the energy $E: \Omega M\left(x_{0}, H_{0}\right) \rightarrow R$ satisfies the Palais-Smale condition.

Proof. Let $\left\{\gamma_{n}\right\}$ be a sequence in $\Omega M\left(x_{0}, H_{0}\right)$ on which $E$ is bounded (say $E\left(\gamma_{n}\right)<k<\infty, n \in Z$ ) and for which $\left\|\operatorname{grad} E_{0}\left(\gamma_{n}\right)\right\|_{0}$ $\rightarrow 0$ as $n \rightarrow \infty$. We want to show that $\left\{\gamma_{n}\right\}$ has a convergent subsequence.

By Proposition 2.9, we can assume w.1.o.g. that

$$
\left\|\operatorname{grad} E\left(\gamma_{n}\right)+\left(\operatorname{grad} H_{a}\left(\gamma_{n}\right), a\right)\right\| \rightarrow 0 \text { as } n \rightarrow \infty \text {. }
$$

Now by Proposition 2.7 we can assume that $\gamma_{n}$ converges to a continuous loop $\gamma$ in the $d_{\infty}$-topology.

From now on we work locally in a natural chart around $\gamma$. Let $N_{\gamma}$ be an open neighborhood of the zero-section such that $\exp _{\gamma}: L^{2}\left(N_{\gamma}\right) \rightarrow$ $\Omega M\left(x_{0}\right)$ is a natural chart around $\gamma$ in $\Omega M\left(x_{0}\right)$, where $L^{2}\left(N_{\gamma}\right)$ is the $L^{2}$-sections of $\gamma^{*} T M$ belonging to $N_{\gamma}$ and vanishing at the end points, i.e., an open neighborhood of the zero section 0 in $T_{\gamma} \Omega M\left(x_{0}\right)$ $=L^{2}\left(\gamma^{*} T M\right)_{0}$.

Let $X_{n}=\exp \left(\gamma_{n}\right)$ so that there is $X_{\infty}$ such that $\left\|X_{n}-X_{\infty}\right\|_{\infty} \rightarrow 0$, as $n \rightarrow \infty$. Using the local expression for the energy (see [14]) it follows that $\left\|X_{n}\right\|$ is bounded. Furthermore, $E$ is locally coercive (see [14])

$$
\begin{gathered}
(d E(x)+(d H(X), a)-(d E(Y)+(d H(Y), a)))(X-Y) \\
\geq \lambda\|X-Y\|-C\|X-Y\|_{\infty}
\end{gathered}
$$

for sufficiently small $\|X\|_{\infty}$ and $\|Y\|_{\infty}$. We have used $E$ (respectively $H$ ) for $E \circ \exp _{\gamma}$ (respectively $H \circ \exp _{\gamma}$ ) which should cause no 
confusion. Now

$$
\begin{aligned}
\lambda\left\|X_{i}-X_{j}\right\| \leq & \left(d E\left(X_{i}\right)+\left(d H\left(X_{i}\right), a\right)\right. \\
& \left.-\left(d E\left(X_{j}\right)+\left(d H\left(X_{j}\right), a\right)\right)\right)\left(X_{i}-X_{j}\right)+c\left\|X_{i}-X_{j}\right\|_{\infty} ;
\end{aligned}
$$

thus $X_{n}$ is a Cauchy sequence and convergent. So $\left\{\gamma_{n}\right\}$ is convergent.

REMARK. The above proof also works for the punctured horizontal loop space $\Omega M\left(x_{0}, e\right) \cap E_{\varepsilon}^{+}$, where $E_{\varepsilon}^{+}$is the space of loops $\gamma$ with $E(\gamma) \geq \varepsilon$ (for any positive number $\varepsilon$ ).

\section{Index theorem.}

3.1. Index theorem on $M$. In this subsection we will prove an index theorem for a quadratic functional defined via a 2 nd order differentialintegral operator subject to a constraint. In particular, the second variation of $E$ on $\Omega M\left(x_{0}, H_{0}\right)$ has the form considered here (cf. Appendix).

The quadratic form in question is $Q^{\tau}(x, x)$, where

$$
\begin{aligned}
Q^{\tau}\left(x_{1}, x_{2}\right)= & \int_{0}^{\tau}\left\{\left(\dot{x}_{1}, \dot{x}_{2}\right)+\left(B(t) \dot{x}_{1}, x_{2}\right)+\left(C x_{1}, x_{2}\right)\right\} d t \\
& +\left\langle b, \int_{0}^{\tau} d t \int_{0}^{t}\left[F(t) x_{1}(t), F(s) x_{2}(s)\right] d s\right\rangle
\end{aligned}
$$

which is defined on the space $H^{\tau}=\left\{x:[0, \tau] \rightarrow R^{m}, x\right.$ continuous and piecewise smooth, $\left.x(0)=x(\tau)=0, \int_{0}^{\tau} F(t) x(t) d t=0\right\}$ where $B, C$ are $m \times m$ matrices: $B^{t}=-B, C^{t}=C$; (, ) is the inner product on $R^{m}$. For $t \in[0, \tau], F(t)$ is a linear map $R^{m} \rightarrow g,[$, is the Lie bracket of $g$, and $\langle$,$\rangle is the dual bracket between g$ and $g^{*}$, and $b \in g^{*}$ is constant.

We always assume that $F(t): R^{m} \rightarrow g$ is a submersion for $t \in$ $[0, \tau]$, which is essentially the fatness assumption (cf. Appendix).

We will also write $Q^{\tau}(x, x)$ as $Q^{\tau}(x)$ for simplicity.

Note we have the formula

$$
\begin{aligned}
Q\left(x_{1}+x_{2}\right)= & Q\left(x_{1}\right)+Q\left(x_{2}\right) \\
& +\int_{0}^{\tau}\left(L x_{1}, x_{2}\right) d t-\sum x_{2}\left(t_{i}\right) \Delta \dot{x}_{1}\left(t_{i}\right)
\end{aligned}
$$

where $L$ is a 2 nd order linear differential-integral operator

$$
\begin{aligned}
(L x)(t)= & -2\left(\partial^{2} x(t) / \partial t^{2}\right)+(B(t) \partial x(t) / \partial t) \\
& +\partial(B(t) x(t)) / \partial t+2 C(t) x(t)+2(F(t))^{*} \operatorname{ad}_{\int_{0}^{t} F(s) x(s) d s^{b}}^{*}
\end{aligned}
$$


and $\Delta \dot{x}(t)$ is the jump of $\dot{x}$ at $t$

$$
\Delta \dot{x}(t)=\dot{x}(t+0)-\dot{x}(t-0)
$$

and the summation in (3.1) is taken over the $t$ where $\dot{x}(t)$ is discontinuous.

Recall that the index of $Q^{\tau}$ is the maximal dimension of subspaces in $H^{\tau}$ on which $Q^{\tau}$ is negative definite.

To compute the index we take a finite dimensional approximation $A^{\tau}$ of $H^{\tau}$ in the following way:

First take a subdivision $0=t_{0}<t_{1}<\cdots<t_{N}=\tau$. Let $A^{\tau}$ be the vector space of such $x$ satisfying

(1) $x$ is continuous on $[0, \tau]$;

(2) $x$ is smooth on $\left(x_{i}, x_{i+1}\right), i=0,1, \ldots, N-1$;

(3) $x(0)=x(\tau)=0$;

(4) $L x=F^{*} c \quad\left(c \in g^{*}\right.$ is constant);

(5) $\int_{0}^{\tau} F(t) x(t) d t=0$.

The construction of $A^{\tau}$ is as follows: Let $e_{1}, \ldots, e_{n}$ be an orthonormal basis in $g^{*}$ for an inner product $\langle,\rangle_{g^{*}}$ on $g^{*}$. Every $x$ in $A^{\tau}$ can be written as

$$
x=u_{1}+b_{1} u^{1}+\cdots+b_{n} u^{n}
$$

where $u_{1}$ satisfies (1), (2) above, and

$$
L u_{1}=0, \quad u_{1}\left(t_{i}\right)=x\left(t_{i}\right), \quad i=1, \ldots, N-1 ;
$$

and $u^{k}$ satisfies (1), (2) above, and

$$
L u^{k}=F^{*}\left(e_{k}\right), \quad u^{k}\left(t_{i}\right)=0, \quad i=0,1, \ldots, N .
$$

Also $b_{1}, b_{2}, \ldots, b_{n}$ are constants satisfying

$$
\int_{0}^{\tau}\left(u_{1}+b_{1} u^{1}+\cdots+b_{n} u^{n}\right) d t=0 \text {. }
$$

Proposition 3.1. Suppose that $t_{i+1}-t_{i}=\tau / N, i=1, \ldots, N-1$. Then if $N$ is sufficiently big, $u_{1}$ is uniquely determined by $x\left(t_{i}\right)$, $i=1, \ldots, N-1$; and $u^{1}, \ldots, u^{n}$ are unique. Moreover, the $n \times n$ matrix

$$
\left(\left\langle\int_{0}^{\tau} F(s) u^{i}(s) d s, e_{j}\right\rangle_{g^{*}}\right)
$$

is non-singular. 
Proof. The first two statements are trivial; we need only to prove the last statement. By a rescaling of each interval $\left[t_{i}, t_{i+1}\right]$, we see that the function $v(t)=u^{k}\left(t \tau / N+t_{i}\right), t \in[0,1]$, satisfies $v(0)=v(1)=0$ and

$$
\frac{d^{2} v(t)}{d t^{2}}=-(\tau / N)^{2} F^{*}\left(e_{k}\right)\left(t_{i}\right)+o(\tau / N)^{2}
$$

hence

$$
u^{k}(t)=F^{*}\left(e_{k}\right)\left(t_{i}\right)\left(t-t_{i}\right)\left(t-t_{i+1}\right)+o(\tau / N)^{2}, \quad t_{i} \leq t \leq t_{i+1},
$$

so

$$
\begin{aligned}
& \left(\left\langle\int_{0}^{\tau} F(s) u^{i}(s) d s, e_{j}\right\rangle_{g^{*}}\right) \\
& \quad=-\frac{\tau^{2}}{6 N^{2}}\left(\int_{0}^{\tau}\left\langle F^{*}(s) e_{i}(s), F^{*}(s) e_{j}(s)\right\rangle_{g^{*}} d s\right)+o(\tau / N)^{2} .
\end{aligned}
$$

Since $F^{*}: g^{*} \rightarrow R^{m}$ is an immersion, the first term above is a nonsingular matrix; hence the proposition follows.

By the above result, we see that the map $\Gamma^{\tau}: A^{\tau} \rightarrow R^{(N-1) m}$

$$
x \rightarrow\left(x\left(t_{1}\right), x\left(t_{2}\right), \ldots, x\left(t_{N-1}\right)\right)
$$

is an isomorphism.

Proposition 3.2. Let the subdivision

$$
0=t_{0}<t_{1}<\cdots<t_{N}=\tau+\varepsilon
$$

be sufficiently small. Then the isomorphism $\Gamma^{\tau+\varepsilon}$ depends smoothly on $\varepsilon$ if $\varepsilon$ is small enough with $t_{1}, \ldots, t_{N-1}$ fixed.

Proof. We need to prove that the solution to

$$
L u=F^{*} c, \quad u(0)=0, \quad u(b)=a
$$

is a continuous function of $b$ for $b \neq 0$ sufficiently small (keeping $F$, $a$ fixed), and this is a well-known fact in the theory of O.D.E.

We will see that $A^{\tau}$ is a "faithful" approximation of $H^{\tau}$.

Proposition 3.3. $H^{\tau}$ splits as a direct sum:

$$
H^{\tau}=A^{\tau}+B^{\tau}
$$


such that

$$
Q^{\tau}\left(x_{1}+x_{2}\right)=Q^{\tau}\left(x_{1}\right)+Q^{\tau}\left(x_{2}\right),
$$

for $x_{1} \in A^{\tau}, x_{2} \in B^{\tau}$, and if the subdivision is small enough, then

$$
Q^{\tau}(x)>0 \text { for } x \in B^{\tau}, \quad x \neq 0 .
$$

(Hence the index of $Q^{\tau}$ on $H^{\tau}$ is that of $Q^{\tau}$ on $A^{\tau}$.)

Proof. $B^{\tau}$ is the space of functions $x \in H^{\tau}$ such that $x\left(t_{i}\right)=0$, $i=1, \ldots, N$. Given any $x \in H^{\tau}$, there is a unique $u \in A^{\tau}$ such that

$$
u\left(t_{i}\right)=x\left(t_{i}\right), \quad i=0,1, \ldots, N .
$$

Clearly $x-u \in B^{\tau}$. Thus the two subspaces $A^{\tau}$ and $B^{\tau}$ generate $H^{\tau}$, and only have the zero function in common.

Then (3.3) follows from (3.1), and we need only to prove the positivity of $Q^{\tau}$ on $B^{\tau}$. Now

$$
\begin{aligned}
Q^{\tau}(x)=\int_{0}^{\tau}\{(\dot{x}, \dot{x}) & +(B \dot{x}, x)+(C x, x) \\
& \left.+\left\langle b, \int_{0}^{\tau} d t \int_{0}^{t}[F(t) x(t), F(x) x(s)] d s\right\rangle\right\} .
\end{aligned}
$$

The last term in the above expression is

$$
\begin{aligned}
\langle b & \left.\int_{0}^{\tau} d t \int_{0}^{t}[F(t) x(t), F(s) x(s)] d s\right\rangle \\
& \geq-M_{0} \int_{0}^{\tau} d t|x(t)| \int_{0}^{t}|x(s)| d s \geq-M_{0}\left(\int_{0}^{\tau}|x(t)| d t\right)^{2} \\
& \geq-M_{1} \int_{0}^{\tau}|x(t)|^{2} d t .
\end{aligned}
$$

So

$$
Q^{\tau}(x) \geq \int_{0}^{\tau}\left\{(\dot{x}, \dot{x})+(B x, \dot{x})+(C x, x)-M|x|^{2}\right\} d t=P^{\tau}(x) .
$$

Now we need only to prove that if $\tau$ is sufficiently small, $P^{\tau}(x) \geq 0$, for $x \in B^{\tau}$, and the equality holds only if $x=0$. Using

$$
\int_{0}^{\tau}\|x(t)\|^{2} d t \leq \tau^{2}\left(\int_{0}^{\tau}\|\dot{x}(t)\|^{2} d t\right) / 2
$$




$$
\begin{aligned}
& P^{\tau}(x)= \int_{0}^{\tau}\left\{(\dot{x}, \dot{x})+(B \dot{x}, x)+(C x, x)-M|x|^{2}\right\} d t \\
& \geq \int_{0}^{\tau}\{(\dot{x}, \dot{x})-2((B \dot{x}, B \dot{x}) / k)+k(x, x) \\
&\left.+(C x, x)-M|x|^{2}\right\} d t \\
& \geq \int_{0}^{\tau}\{(1-(2\|B\| / k))(\dot{x}, \dot{x})-(2 k+\|C\|+M)(x, x)\} d t \\
& \geq \int_{0}^{\tau}\left\{(1-(2\|B\| / k)) / \tau^{2}-(2 k+\|C\|+M)\right\}(x, x) d t
\end{aligned}
$$

where $\|B\|=\max \|B(t)\|,\|C\|=\max \|c(t)\|$, and $k$ is taken to be $k=4\|B\|$. Then when $\tau$ is sufficiently small,

$$
\begin{gathered}
d=1 /\left(2 \tau^{2}\right)-4\|B\|-\|C\|-M>0 \\
P^{\tau}(x) \geq d \int_{0}^{\tau}(x, x) d t \geq 0
\end{gathered}
$$

and the equality only holds for $x=0$.

Now the computation of the index of $Q^{\tau}$ on $H^{\tau}$ reduces to that of $Q^{\tau}$ on the finite-dimensional space $A^{\tau}$. Next we define the notion of conjugate points as in Riemannian geometry.

Definition 3.1. We say that $\tau_{1}$ is conjugate to 0 if there is a smooth function $x:\left[0, \tau_{1}\right] \rightarrow R^{m}$ satisfying

(1) $L x=F^{*} c \quad\left(c \in g^{*}\right.$ is constant $)$;

(2) $x(0)=x\left(\tau_{1}\right)=0$;

(3) $\int_{0}^{\tau_{1}} F(t) x(t) d t=0$.

If $\tau_{1}$ is conjugate to 0 , then its multiplicity is the maximal number of linearly independent $x$ satisfying (1), (2), (3).

Now we state the index theorem.

THEOREM 3.1. The index of $Q^{\tau}$ on $H^{\tau}$ is equal to the number of conjugate points to 0 in $[0, \tau)$, counted together with their multiplicity.

Proof. We will prove the theorem in four steps.

Step 1 . Let $\mu(\tau)$ be the index of $Q^{\tau}$ on $H^{\tau}$. Then for $\tau$ sufficiently. small, $\mu(\tau)=0$.

The proof of this is similar to that of Proposition 3.3 and we will not repeat.

Step 2. Let $\tau<\tau^{\prime}$; then $\mu(\tau) \leq \mu\left(\tau^{\prime}\right)$. 
In fact, by Definition 3.1, there is a subspace of $H^{\tau}, V$ of dimension $\mu(\tau)$ such that $Q^{\tau}$ is negative definite on $V$. Extend $x \in V$ by zero; then we can consider $V$ as a subspace of $H^{\tau^{\prime}}$. So $\mu\left(\tau^{\prime}\right) \geq \mu(\tau)$.

Step 3. If $\tau$ is not conjugate to 0 , then for $\varepsilon$ sufficiently small, $\mu(\tau+\varepsilon)=\mu(\tau)$.

If $\tau$ is conjugate to 0 with multiplicity $k$, then

$$
\mu(\tau+\varepsilon) \leq \mu(\tau)+k .
$$

We take subdivison $0=t_{0}<t_{1}<\cdots<t_{N}=\tau$. According to Proposition 3.3, $\mu(\tau)$ is the index of $Q^{\tau}$ on $A^{\tau}$, which is isomorphic to $R^{(N-1) m}$. So we have a one-parameter family of quadratic forms (which we still denote by $Q^{\tau}$ ) on $R^{(N-1) m}$ depending smoothly on $\tau$. Then $Q^{\tau}$ is positive definite on a subspace of codimension $\mu(\tau)+k$ in $R^{(N-1) m}$, and so is $Q^{\tau+\varepsilon}$ if $\varepsilon$ is small enough. So $\mu(\tau+\varepsilon) \leq \mu(\tau)+\varepsilon$.

Step 4 . We want to prove that if $\tau$ is conjugate to 0 with multiplicity $k$, then $\mu(\tau+\varepsilon) \geq \mu(\tau)+k$.

Now there are linearly independent $w_{1}, \ldots, w_{\mu(\tau)} \in H^{\tau}$ such that $Q^{\tau}$ is negative on them.

Assume w.l.o.g. that $u_{1}, \ldots, u_{c} \in H^{\tau}$ (respectively $v_{1}, \ldots, v_{d} \in$ $\left.H^{\tau}, c+d=k\right)$ such that

$$
L u_{i}=F^{*} c_{i}, \quad i=1, \ldots, c ;
$$

and $\dot{u}_{1}(\tau), \ldots, \dot{u}_{c}(\tau)$ are linearly independent (respectively $L v_{i}=$ $\left.F^{*} e_{i}, \dot{v}_{i}(\tau)=0, i=1, \ldots, d\right)$. Then there are $x_{1}, \ldots, x_{c} \in H^{\tau+\varepsilon}$, such that the $c \times c$ matrix

$$
\left(\left(\dot{u}_{i}(\tau), x_{j}(\tau)\right)\right.
$$

is the identity matrix. Also there are $y_{1}, \ldots, y_{d} \in H^{\tau+\varepsilon}$ such that the $d \times d$ matrix

$$
\left(\int_{0}^{\tau}\left(F^{*} e_{i}, y\right) d t\right)
$$

is the identity matrix, and $y_{i}(\tau)=0, i=1, \ldots, d$.

From this and (3.2) we immediately get

$$
\begin{array}{ll}
Q^{\tau+\varepsilon}\left(u_{i}, x_{j}\right)=-\delta_{i j}, & i=1, \ldots, c ; j=1, \ldots, c ; \\
Q^{\tau+\varepsilon}\left(v_{i}, y_{j}\right)=-\delta_{i j}, & i=1, \ldots, d, j=1, \ldots, d ; \\
Q^{\tau+\varepsilon}\left(u_{i}, y_{j}\right)=Q^{\tau+\varepsilon}\left(v_{j}, x_{i}\right)=0, & i=1, \ldots, c, j=1, \ldots, d .
\end{array}
$$

Now $Q^{\tau+\varepsilon}$ is negative on $w_{1}, \ldots, w_{\mu(\tau)}, \frac{1}{\nu} u_{1}+\nu x_{1}, \ldots, \frac{1}{\nu} u_{c}+\nu x_{c}$, $\frac{1}{\nu} v_{1}+\nu y_{1}, \ldots, \frac{1}{\nu} v_{d}+\nu y_{d}$ for sufficiently small $\nu$. In fact, the 
compound matrix of $Q^{\tau+\varepsilon}$ with respect to these vector fields is

$$
\left|\begin{array}{cc}
\left(Q^{\tau}\left(w_{i}, w_{j}\right)\right) & \nu C \\
\nu D & -I+\nu^{2} B
\end{array}\right|
$$

where $B, C, D$ are matrix independent of $\nu$. Obviously, if $\nu$ is sufficiently small, the above matrix is negative definite. Hence $\mu(\tau+\varepsilon) \geq \mu(\tau)+c+d=\mu(\tau)+k$.

3.2. Index Theorem on $F$. We now come to the index theorem for Problem 1.

Suppose that $\gamma_{0} \in \Omega F\left(x_{1}, x_{2}\right)$ is a critical point of $E$ on $\Omega F\left(x_{1}, x_{2}\right)$. The Morse index of $E$ at $\gamma_{0}$ is the maximal dimension of subspaces of $T_{\gamma_{0}} \Omega F\left(x_{1}, x_{2}\right)$ on which the quadratic differential $E_{* *}$ is negative definite. We want to express the Morse index in terms of the conjugate points as in Riemannian geometry.

Definition 3.2. We way that $x_{2}$ is conjugate to $x_{1}$ along $\gamma_{0}$ if there is a smooth one-parameter family of horizontal paths $\gamma_{\varepsilon} \in$ $\Omega F\left(x_{1}^{\varepsilon}, x_{2}^{\varepsilon}\right)$ such that for each $\varepsilon, \gamma_{\varepsilon}$ is a horizontal extremal of $E$ on $\Omega F\left(x_{1}^{\varepsilon}, x_{2}^{\varepsilon}\right)$, and the vector field on $\gamma_{0}$

$$
\partial\left(\gamma_{\varepsilon}\right) /\left.\partial \varepsilon\right|_{\varepsilon=0}
$$

is not identically zero and vanishes at the end points $x_{1}, x_{2}$. The index of $x_{2}$ along $\gamma_{0}$ is the maximal number of one-parameter families of horizontal extremals $\gamma_{\varepsilon}^{1}, \ldots, \gamma_{\varepsilon}^{m}$, such that the vector fields along $\gamma_{0}$

$$
\partial\left(\gamma_{\varepsilon}^{1}\right) /\left.\partial \varepsilon\right|_{\varepsilon=0}, \ldots ; \partial\left(\gamma_{\varepsilon}^{m}\right) /\left.\partial \varepsilon\right|_{\varepsilon=0}
$$

are linear independent and vanish at the end points $x_{1}, x_{2}$.

THEOREM 3.2. Suppose that the bundle is fat and $\gamma_{0}$ is an extremal of $E$ on $\Omega F\left(x_{1}, x_{2}\right)$. Then the Morse index of $E$ on $\Omega F\left(x_{1}, x_{2}\right)$ at $\gamma_{0}$ is the number of conjugate points to $x_{1}$ along $\gamma_{0}$, counted together with their multiplicity ( $x_{2}$ should not be counted).

Proof. Project $\gamma_{0}$ down to a path $\gamma_{1}$ on $M$. Then the Morse index of $E$ at $\gamma_{0}$ is just that of the quadratic form defined via the 2 nd order differential-integral operator (cf. Appendix)

$$
\begin{aligned}
L X= & D^{2} X / d t^{2}+R(V, X) V-Q(t)\left(\nabla_{J} F_{\omega}\right)(X,) \\
& -Q(t) F_{\omega}(D X / d t,) \\
& -U(t) \operatorname{ad}_{\int_{0}^{t} U F_{\omega}(V, X) U^{-1} d s}^{*} Q(0) F_{\omega}(V,) U^{-1}(t)
\end{aligned}
$$


(where $V(t)=\dot{\gamma}(t)$ ) defined on the subspace of vector fields along $\gamma_{0}$ :

$$
H=\left\{X, \int_{0}^{1} U F_{\omega}(V, X) U^{-1} d t=0\right\} .
$$

By Theorem 3.1, the index of the quadratic form is just the number of conjugate points (in the sense of Definition 3.1) to $\gamma_{1}(0)$ along $\gamma_{1}$, counted together with their multiplicity. So we only need to prove that $\gamma_{0}(\tau)$ is conjugate to $\gamma_{0}(0)$ with multiplicity $m$ along $\gamma_{0}$ (in the sense of Definition 3.2) if and only if $\gamma_{1}(\tau)=\pi \gamma_{0}(\tau)$ is conjugate to $\gamma_{1}(0)$ with multiplicity $m$ along $\pi \gamma_{0}$ (in the sense of Definition 3.1). Obviously, if a family of horizontal extremals $\gamma_{\varepsilon}$ on $F$ is such that $X_{0}=\partial \gamma /\left.\partial \varepsilon\right|_{\varepsilon=0} \neq 0$, then $X=\pi_{*} \partial \gamma /\left.\partial \varepsilon\right|_{\varepsilon-0} \neq 0$ satisfies $L X=Q U F_{\omega}(V,) U^{-1}$, and if $X_{0}$ vanishes at the end points, then $X$ satisfies

$$
\int_{0}^{1} U F_{\omega}(V, X) U^{-1} d t=0
$$

We need only to show that every $X$ along $\gamma_{1}$ satisfying $L X-$ $Q U F_{\omega}(V,) U^{-1}$, and if $X_{0}$ vanishes at the end points, then $X$ satisfies

$$
\int_{0}^{1} U F_{\omega}(V, X) U^{-1} d t=0
$$

We need only to show that every $X$ along $\gamma_{1}$ satisfying

$$
L X=Q U F_{\omega}(V,) U^{-1}
$$

can be given in this way: there is a family of horizontal extremals $\gamma_{\varepsilon}$ such that $X=\pi_{*} \partial \gamma /\left.\partial \varepsilon\right|_{\varepsilon=0}$. To see this, define map $e_{x}: T_{x} F \rightarrow F$ by

$$
e_{x}(v)=\exp (-\omega(y)) \exp _{x}(v)
$$

where $v=y+z \in T_{x} F, y$ is tangent to the fiber, $z$ is horizontal, $\exp _{x}: T_{x} F \rightarrow F$ is the exponential map of the Kaluza-Klein metric on $F$, and exp is the exponential map of $G$. We will write $e$ for $e_{x}$ for simplicity. Then $\gamma_{0}$ can be written as $t \rightarrow e\left(t v_{0}\right)$ (Montgomery [18]) for some $v \in T-x F$. Moreover, for $t_{0}$ sufficiently small, $e_{*}$ is regular at $v_{0} t_{0}$ (this will be proved in $\S 3.3$ ). So there is a family of $v_{\varepsilon}$ depending smoothly on $\varepsilon$, such that the differential of $e$ at $t_{0} v_{0}$,

$$
e_{*}\left(v_{\varepsilon}\right)=\int_{0}^{t_{0}} U F_{\omega}(V,) U^{-1} d s e\left(t_{0} v_{0}\right)+X_{1}
$$

where $X_{1}$ is horizontal satisfying $\pi_{*} X_{1}=X\left(t_{0}\right)$. Now define a family 
of horizontal extremals $\gamma_{\varepsilon}$ by

$$
\gamma_{\varepsilon}(t)=e\left(t v_{\varepsilon}\right)
$$

Then $\pi_{*} \partial \gamma /\left.\partial \varepsilon\right|_{\varepsilon=0}=X$. Hence the theorem is proved.

3.3. The existence of non-conjugate points. Fixing $x_{0} \in F$, we will prove that almost every point on $F$ is not conjugate to $x_{0} \in F$ along any horizontal extremals.

Let $e_{x}: T_{x} F \rightarrow F$ be the map defined by (3.5). It is known that for $v \in T_{x} F, \gamma(t)=e x(t v)$ is an extremal of the energy $E$ on $\Omega F\left(x, x_{1}\right)$, where $x_{1}=e_{x}(v)$. Conversely, if the bundle is fat, then every extremal $\gamma$ of $E$ on $\Omega F\left(x, x_{1}\right)$ can be written in the form $\gamma(t)=e_{x}(t v)$ for some $v \in T-x F$ (cf. [18], [20]).

Proposition 3.4. Suppose that $F \rightarrow M$ is a fat bundle. Then $x_{1}=$ $e_{x}(v)$ is conjugate to $x$ (with $x \neq x_{1}$ ) along $\gamma(t)=e_{x}(t v)$ if and only if the map $e_{x}$ is singular at $v$.

Proof. Suppose that $e_{x}$ is singular at $v_{0} \in T_{x} F$. Then $e_{x^{*}}\left(v_{1}\right)=0$ for some non-zero $v_{1} \in T_{x} F$.

Let $s \rightarrow v(s)$ be a path in $T_{x} F$ such that $v(0)=v_{0}$, and $d v(0) /\left.d s\right|_{s=0}=v_{1}$. Then the one-parameter family of paths defined by

$$
\gamma_{s}(t)=e_{x}(t v(s))
$$

is a variation through geodesics of the geodesic $\gamma_{0}$. Therefore the vector field $W$ given by $t \rightarrow \partial\left(e_{x}(t v(s))\right) /\left.\partial S\right|_{s=0}$ is an extremal field along $\gamma_{0}$. Obviously $W(0)=0$. We also have

$$
W(1)=e_{x}(v(s)) /\left.\partial s\right|_{s=0}=e_{x^{*}}\left(v_{1}\right)=0 .
$$

Now, we need only to show that $W$ is not identically zero. If $W=0$, then (we write $v_{1}=y+z, y$ is tangent to the fiber, $z$ is horizontal)

$$
D W /\left.d t\right|_{t=0}=z
$$

is zero, and also

$$
D^{2}\left(\pi_{*} W\right) /\left.d t^{2}\right|_{t=0}=\left(y, F_{\omega}\left(v_{0}, \cdot\right)\right)=0 .
$$

Since the bundle is fat, either $z=0, y=0, v_{1}=y+z=0$, or $v_{0}=0$ in which case $x_{1}=x$. Either case is a contradiction.

Using Sard's theorem, we get the following result, which has been used in the proof of Theorem 3.2. 
COROLlaRY 3.1. Fixing $x_{0} \in F$, then for almost every point $x_{1}$ of $F, x_{1}$ is not conjugate to $x_{0}$ along any extremals.

3.4. The existence of arbitrary nearby conjugate points. In [20] it is proved that for $x \in F$, there are cut points arbitrarily near $x$. In this subsection we prove a slightly stronger result:

PROPOSITION 3.5. There are conjugate points arbitrarily near $x$.

Proof. Assume the contrary. Consider the sphere $B_{\varepsilon}=\{y: d(y, x)$ $=\varepsilon\}$ and the set $e_{x}^{-1}\left(B_{\varepsilon}\right)$ in $T_{x} F$, where $d$ is the Carnot-Caratheodory distance on $F$. Let $H_{\varepsilon}=e_{x}^{-1}\left(B_{\varepsilon}\right) \cap\left\{v \in T_{x} F,\left|\pi_{*} v\right|=\varepsilon\right\}$. Then the map

$$
e_{x}: H_{\varepsilon} \rightarrow B_{\varepsilon}
$$

is an onto map. And it is everywhere regular, hence is a covering map. But $H_{\varepsilon}$ is a set in the cylinder $S^{m} \times R^{n}$, and it is impossible for a subset in the cylinder to cover the sphere $S^{m+n}$, hence a contradiction.

\section{Horizontal loop spaces.}

4.1. An abstract model. Let $l^{2}$ be the Hilbert space

$$
l^{2}=\left\{x=\left(a_{1}, a_{2}, \ldots\right),|x|=\left(\sum\left|a_{i}\right|^{2}\right)^{1 / 2}\right\}
$$

and $P l^{2}$ is projection. Suppose that we have a smooth map $f: l^{2} \rightarrow$ $R^{n}$, where $f=f_{0}+f_{1}$ satisfies

(1) $f_{0}=\left(f_{1}^{0}, \ldots, f_{n}^{0}\right)$ is a quadratic map and a submersion everywhere except at $x=0 \in l^{2}$, i.e. for $\left(b_{1}, \ldots, b_{n}\right) \in R^{n}-\{0\}$, $x \neq 0$

$$
b_{1} d f_{1}^{0}(x)+\cdots+b_{n} d f_{n}^{0}(x) \neq 0
$$

(2) $f_{1}$ consists of higher terms

$$
\left|f_{1}(x)\right| \leq M|x|^{3} ;
$$

(3) $f$ is a submersion except at $x=0 \in l^{2}$.

The set $f^{-1}(0)$ is smooth everywhere except at 0 , and 0 is the singularity. In this subsection we will resolve this singularity.

As in the finite dimensional case, we define the local charts for $\mathrm{Pl}^{2}$ to be

$$
\begin{gathered}
\phi_{i}: l^{2} \rightarrow P l^{2}, \\
\phi_{i}\left(\alpha_{1}, \ldots, \alpha_{i-1}, \alpha_{i+1}, \ldots\right)=\left\{\alpha_{1}, \ldots, \alpha_{i-1}, 1, \alpha_{i+1}, \ldots\right\},
\end{gathered}
$$


and transition maps are

$$
\begin{aligned}
& \phi_{j}^{-1} \circ \phi_{i}\left(\alpha_{1}, \ldots, \alpha_{i-1}, \alpha_{i+1}, \ldots\right) \\
& \quad=\left(\alpha_{1} / \alpha_{j}, \ldots, \alpha_{i-1} / \alpha_{j}, 1 / \alpha_{j}, \alpha_{i+1} / \alpha_{j}, \ldots\right) \quad\left(\alpha_{j} \neq 0\right) .
\end{aligned}
$$

Note that $\Phi_{j}^{-1} \circ \Phi_{i}$ can be written as

$$
\Phi_{j}^{-1} \circ \Phi_{i}=a b
$$

where $b$ is a map $b: l^{2} \rightarrow l^{2}$

$$
b\left(\alpha_{1}, \alpha_{2}, \ldots\right)=\left(\alpha_{1}, \ldots, \alpha_{i-1}, 1, \alpha_{i+1}, \ldots\right)
$$

and $a: l^{2} \cap\left\{\alpha_{j} \neq 0\right\} \rightarrow R$

$$
a\left(\alpha_{1}, \alpha_{2}, \ldots\right)=1 / \alpha_{j} .
$$

Since $a$ and $b$ are smooth, so is $\Phi_{j}^{-1} \circ \Phi_{i}$.

Let $B: B l^{2} \rightarrow l^{2}$ be the real blow-up of $l^{2}$ at 0 . Recall that $B l^{2}$ is the set in $P l^{2} \times l^{2}$ defined by the quadratic relations:

$$
\begin{array}{r}
B l^{2}=\left\{\left(\left\{\alpha_{1}, \alpha_{2}, \ldots\right\},\left(\beta_{1}, \beta_{2}, \ldots\right)\right) \in P l^{2} \times l^{2},\right. \\
\left.\alpha_{i} \beta_{j}=\alpha_{j} \beta_{i}, i, j=1,2, \ldots\right\}
\end{array}
$$

and $B$ is the restriction of the projection pr: $P l^{2} \times l^{2} \rightarrow l^{2}$ to $B l^{2}$.

Definition 4.1. Define the blow-up of $f^{-1}(0), B_{f=0}$, to be the closure of the set $B^{-1}\left(f^{-1}(0)-\{0\}\right)$ in $B l^{2}$, and $B^{f}$ to be the restriction of $B$ to $B_{f=0}$

$$
B^{f}: B_{f=0} \rightarrow f^{-1}(0) .
$$

THEOREM 4.1. (1) $B_{f=0}$ is a smooth manifold;

(2) $B^{f}: B^{-1}\left(f^{-1}(0)-\{0\}\right) \rightarrow f^{-1}(0)-\{0\}$ is a diffeomorphism;

(3) $\left(B^{f}\right)^{-1}(0)$ is an embedding of a projective variety into $B_{f=0}$ as a submanifold of codimension one. This projective variety is defined by $f_{0}(x)=0$.

Proof. (1) Let $\left(\left\{\alpha_{1}, \alpha_{2}, \ldots\right\},\left(\beta_{1}, \beta_{2}, \ldots\right)\right)$ be the coordinates on $P l^{2} \times l^{2}$. Define a map $A$ on $\left(P l^{2} \cap\left\{\alpha_{1} \neq 0\right\}\right) \times l^{2}$ by means of the local chart $\Phi_{1}$ in (4.3):

$$
\begin{aligned}
& A:\left(P l^{2} \cap\left\{\alpha_{1} \neq 0\right\}\right) \times l^{2} \rightarrow l_{\infty} \times R^{n}, \\
& \begin{array}{l}
A\left(\left\{1, \alpha_{2}, \alpha_{3}, \ldots\right\},\left(\beta_{1}, \beta_{2}, \ldots\right)\right) \\
\quad=\left(\left(\beta_{2}-\alpha_{2} \beta_{1}, \beta_{3}-\alpha_{3} \beta_{1}, \ldots\right),\right. \\
\left.\quad\left(f_{0}\left(1, \alpha_{2}, \alpha_{3}, \ldots\right)+f_{1}\left(\beta_{1}, \alpha_{2} \beta_{1}, \alpha_{3} \beta_{1}, \ldots\right) / \beta_{1}^{2}\right)\right)
\end{array}
\end{aligned}
$$


where $l_{\infty}$ is the Banach space

$$
l_{\infty}=\left\{x=\left(c_{1}, c_{2}, \ldots\right),|x|=\max \left|c_{i}\right|<\infty\right\} .
$$

Obviously $A$ is smooth and submersive, so $A^{-1}(0)$ is a smooth manifold. Note that

$$
A^{-1}(0) \cap\left\{\left(\beta_{1}, \beta_{2}, \ldots\right) \neq 0\right\}=B^{-1}\left(f^{-1}(0)-\{0\}\right) \cap\left\{\alpha_{1} \neq 0\right\},
$$

and $A^{-1}(0) \cap\left\{\left(\beta_{1}, \beta_{2}, \ldots\right)=0\right\}$ is a submanifold of codimension 1 in $A^{-1}(0)$. Hence after taking the closure of $B^{-1}\left(f^{-1}(0)-\{0\}\right)$, we have

$$
A^{-1}(0)=B_{f=0} \cap\left\{\alpha_{1} \neq 0\right\} .
$$

So $B_{f=0}$ is smooth.

(2) This follows from the fact that $B: B l^{2}-B^{-1}\{0\} \rightarrow l^{2}-\{0\}$ is a diffeomorphism.

(3) Obviously

$$
\begin{aligned}
& \left(B^{f}\right)^{-1}(0) \cap\left\{\alpha_{1} \neq 0\right\} \\
& \quad=\left\{\left(\left\{1, \alpha_{2}, \ldots\right\},(0,0, \ldots)\right) \in P l^{2} \times l^{2} f_{0}\left(1, \alpha_{2}, \ldots\right)=0\right\} ;
\end{aligned}
$$

hence $\left(B^{f}\right)^{-1}(0)$ is diffeomorphic to the projective variety:

$$
f_{0}\left(\alpha_{1}, \alpha_{2}, \ldots\right)=0 \text {. }
$$

Intersecting $f^{-1}(0)$ with the $\varepsilon$-sphere $S_{\varepsilon}=\left\{x \in l^{2},|x|=\varepsilon\right\}$, we get

$$
M_{\varepsilon}=f^{-1}(0) \cap S_{\varepsilon} .
$$

Then $T_{\varepsilon}=\left(B^{f}\right)^{-1}\left(\bigcup_{\rho \leq \varepsilon} M_{\varepsilon}\right)$ is a tubular neighborhood of $\left(B^{f}\right)^{-1}(0)$ with boundary $\left(B^{f}\right)^{-1}\left(M_{\varepsilon}\right)$. Note that for sufficiently small $\varepsilon$, the function

$$
h: B_{f=0} \rightarrow R, \quad h(y)=\left|B^{f} y\right|^{2}
$$

has no critical points in $T_{\varepsilon}$ other than $\left(B^{f}\right)^{-1}(0)$, takes the minimal value on $\left(B^{f}\right)^{-1}(0)$, and is constant on $\left(B^{f}\right)^{-1}\left(M_{\varepsilon}\right)$, so we get

Proposition 4.1. For sufficiently small $\varepsilon, m_{\varepsilon}$ is a double covering of $\left(B^{f}\right)^{-1}(0)$.

REMARK. The above arguments also show that $\cup_{\rho \leq \varepsilon} M_{\rho}$ is contractible.

Next we consider $g^{-1}(0)$, where $g$ is a smooth function

$$
g:[0,1]^{m} \times l^{2} \rightarrow R^{n}, \quad g=g_{0}+g_{1} \text {. }
$$

(1) $g(t, 0)=0$ and $g$ is submersive except at $(t, 0) \in[0,1]^{m} \times l^{2}$. 
(2) For fixed $t \in[0,1]^{m}, g_{0}(t, x), g_{1}(t, x)$ satisfy (4.1), (4.2) respectively.

$g^{-1}(0)$ is smooth except at $[0,1]^{m} \times\{0\}$, and we are going to blow up $g^{-1}(0)$ along $[0,1]^{m} \times\{0\}$.

Let $\bar{B}:[0,1]^{m} \times B l^{2} \rightarrow[0,1]^{m} \times l^{2}$ be the blow-up along $[0,1]^{m} \times$ $\{0\}$.

Definition 4.2. Define the blow-up of $g^{-1}(0)$ along $[0,1]^{m} \times\{0\}$, $B_{g=0}$, to be the closure of $(\bar{B})^{-1}\left(g^{-1}(0)-[0,1]^{m} \times\{0\}\right)$ in $[0,1]^{m} \times$ $P l^{2}$, and $B^{g}$ be the restriction of $\bar{B}$ to $B_{g=0}$

$$
B^{g}: B_{g=0} \rightarrow g^{-1}(0) \text {. }
$$

Proposition 4.2. (1) $B_{g=0}$ is a smooth manifold;

(2) $B^{g}: B_{g=0}-\left(B^{g}\right)^{-1}\left([0,1]^{m} \times\{0\}\right) \rightarrow g^{-1}(0)-\left([0,1]^{m} \times\{0\}\right)$ is a diffeomorphism;

(3) $\left(B^{g}\right)^{-1}\left([0,1]^{m} \times\{0\}\right)$ is an embedding of $\left\{(t, x) \in[0,1]^{m} \times\right.$ $\left.P l^{2}, g_{0}(t, x)=0\right\}$ into $B_{g=0}$ as a submanifold of codimension 1 .

The proof is the same as that of Theorem 4.1.

4.2. Horizontal loop spaces. We first study the singularity of the based horizontal loop space $\Omega F\left(x_{0}, x_{0}\right)$ using the results of $\S 4.1$. Then we study the free horizontal loop space. From now on we assume that $F$ is a fat bundle.

Since the singularity is of local character, we choose local coordinates on $F$ and may identify $\bar{F}\left(x_{0}, x_{0}\right)$ with the subset $\bar{R}^{m}(0, e)$ in $\bar{R}^{m}(0)$, satisfying $H(\gamma)=e$; here $\bar{R}^{m}(0)$ is the Hilbert space

$$
\begin{aligned}
\left\{\gamma:[0,1] \rightarrow R^{m}, \gamma(0)=\gamma(1)\right. & =0 \\
& \left.\|\gamma\|=\left(\int_{0}^{1}\left(|\gamma|^{2}+|\dot{\gamma}|^{2}\right) d t\right)^{1 / 2}<\infty\right\} .
\end{aligned}
$$

In terms of the local coordinates, we can compute the Hessian of the holonomy map at the constant loop 0.

Proposition 4.3. $\left(d^{2} H(0),(u, v)\right)=\int_{0}^{1} F_{\omega 0}(u, \dot{v}) d t$ where $F_{\omega 0}$ is the curvature at $0 \in M$.

There is a two-step nilpotent Lie algebra which is independent of the local coordinates. This construction is well known to those working in the subelliptic operators (cf. [20], [22]). 
Definition 4.3. $n_{0}=V_{1}+V_{2}$ is the graded two step nilpotent Lie algebra defined by

$$
\begin{aligned}
& (x, y) \in V_{1} \times V_{1} \rightarrow F_{\omega 0}[x, y] \in V_{2} \\
& (x, y) \in V_{1} \times V_{2} \rightarrow 0 \\
& (x, y) \in V_{2} \times V_{2} \rightarrow 0
\end{aligned}
$$

Now we see that $H$ can be written as

$$
H(\gamma)=t_{0}^{1} F_{\omega 0}(\gamma, \dot{\gamma}) d t+b(\gamma)
$$

where $b$ is a smooth map $b: \Omega R^{m}(0) \rightarrow g$ satisfying

$$
|b(\gamma)|<M\|\gamma\|^{3} \text {. }
$$

Since the bundle $F$ is fat, the quadratic function $\int_{0}^{1} F_{\omega 0}(\gamma, \dot{\gamma}) d t$ satisfies (4.1). Note that $l^{2}$ is isomorphic to $\Omega R^{m}(0)$, applying Theorem 4.1 and Proposition 4.1, we get

THEOREM 4.2. There is a smooth map $B^{H}$ and a smooth manifold $B_{H=e}$, called the blow-up of $\Omega M(0, e)$,

$$
B^{H}: B_{H=e} \rightarrow \Omega M(0, e)
$$

such that

(1) $B^{H}: B_{H=e}-\left(B^{H}\right)^{-1}\{0\} \rightarrow \Omega M(0, e)-\{0\}$ is a diffeomorphism;

(2) $\left(B^{H}\right)^{-1}(0)$ is an embedding of the projective variety $P_{F, 0}$ defined by

$$
\int_{0}^{1} F_{\omega 0}(\gamma, \dot{\gamma}) d t=0
$$

into $B_{H=e}$ as a submanifold of codimension 1 .

(3) Let $m_{\varepsilon}=\Omega M(0, e) \cap\{\gamma: E(\gamma)=\varepsilon\}$. Then for $\varepsilon$ sufficiently small, $M_{\varepsilon}$ is a double covering of $\left(B^{H}\right)^{-1}(0)$.

REMARK. If $N_{x_{0}}$ is Lie algebra isomorphic to $n_{x_{1}}$, then $P_{F, x_{0}}$ differs from $P_{F, x_{1}}$ by an affine transformation. In particular, this shows that the blow-up is independent of the local coodinates.

Now we turn to the free horizontal loop space on $F$, denoted by $\Omega_{H} F$. It is smooth except at constant loops. We want to resolve the singularities. 
Let $F \subset \Omega_{H} F$ be embedded as constant loops. We blow up $\Omega_{H} F$ along $F$ as in Proposition 4.2. Then we get

Proposition 4.4. There is a blow-up of $\Omega_{H} F$ along $F$, denoted by $B \Omega_{H} F$,

such that

$$
B_{H}: B \Omega_{H} F \rightarrow \Omega_{H} F
$$

(1) $B_{H}, B \Omega_{H} F$ are smooth;

(2) $B_{H}: B \Omega_{H} F-B_{H}^{-1}(F) \rightarrow \Omega_{H} F-F$ is a diffeomorphism;

(3) $B_{H}^{-1}(F)$ is an embedded submanifold of codimension 1 in $B \Omega_{H} F$.

To describe $B_{H}^{-1}(F)$, we look at the free loop space $\Omega F$, and the normal bundle of $F$ in $\Omega F$, denoted by $n_{F}$. The fibers of $N_{F}$ are isomorphic to the vector space $\Omega R^{m+n}(0)$. Let the multiplicative group $R-\{0\}$ act on $\Omega R^{m+n}(0)=\Omega R^{m}(0) \times \Omega R^{n}(0)$ by

$$
\left(t,\left(\gamma_{1}, \gamma_{2}\right)\right) \rightarrow\left(t \gamma_{1}, t^{2} \gamma_{2}\right)
$$

and $P_{w} \Omega R^{m+n}(0)$ be the quotient space. Let $P_{w} N_{F}$ be the corresponding quotient bundle of $N_{F}$. Then $B_{H}^{-1}(F)$ can be written as

$$
\begin{aligned}
\left\{\left(z,\left\{\gamma_{1}, \gamma_{2}\right\}\right) \in P_{w} N_{F}, \gamma_{2}(t)=\int_{0}^{t} F_{\omega z}\left(\gamma_{1}, \dot{\gamma}_{1}\right) d s\right. & \\
& \left.\int_{0}^{1} F_{\omega z}\left(\gamma_{1}, \dot{\gamma}_{1}\right) d s=0\right\} .
\end{aligned}
$$

REMARK. Take local coordinates on $F$. Then locally $B_{H}^{-1}(F)$ can be written as

$$
\left\{(z, H,\{\gamma\}) \in U \times G \times P \Omega R^{m}(0), \int_{0}^{1} F_{\omega z}(\gamma, \dot{\gamma}) d s=0\right\},
$$

which is isomorphic to

$$
\begin{aligned}
& \left\{(z, H,\{\gamma\}) \in U \times G \times P H^{1}\left(S^{1}, R^{m}\right),\right. \\
& \left.\qquad \int_{0}^{1} \gamma d s=0, \int_{0}^{1} F(\gamma, \dot{\gamma}) d s=0\right\} .
\end{aligned}
$$

Taking the Fourier series, then the above space is isomorphic to

$$
\begin{aligned}
& \left\{\left(z, H,\left\{\alpha_{1}, \alpha_{2}, \ldots\right\}\right) \in U \times G \times P l^{2},\right. \\
& \left.\quad \sum_{k \text { odd }}^{\infty} F_{\omega z}\left(\left(\alpha_{k n+1}, \ldots, \alpha_{k n+n}\right),\left(\alpha_{k n+n+1}, \ldots, \alpha_{k n+2 n}\right)\right)=0\right\} .
\end{aligned}
$$


Then $B_{H}^{-1}(F)$ is the total space of a fiber bundle over $F$ if $\operatorname{dim}(G)=$ 1. $B_{H}^{-1}(F)$ might still be the total space of a fiber bundle over $F$ in general, but we are not able to prove this.

Let $\widetilde{M}_{\varepsilon}$ be the set of those free horizontal loops $\gamma$ with $E(\gamma)=\varepsilon$; then using the same method as in Proposition 4.1, we arrive at

Proposition 4.5. For $\varepsilon$ sufficiently small, $\widetilde{M}_{\varepsilon}$ is a double covering of $B_{H}^{-1}(F)$.

The $S^{1}$ action on $\Omega_{H} F$ (rotate the loops) lifts to $B \Omega_{H} F-B_{H}^{-1}(F)$ then extends smoothly to $B \Omega_{H} F$.

Proposition 4.6. There is a smooth $S^{1}$-action on $B \Omega_{H} F$ with respect to which the blow-up map $B_{H}$ is equivariant.

Proof. It suffices to show that the action of $S^{1}$ on $B \Omega_{H} F-B_{H}^{-1}(F)$ extends smoothly to $B_{H}^{-1}(F)$.

We take local coordinates on $F$. Then locally $B \Omega_{H} F$ is a smooth submanifold of $G \times\left(\Omega R^{m} / \sim\right) \times \Omega R^{m}$. Here $\Omega R^{m} / \sim$ is the quotient space of $\Omega R^{m}$ (the space of free $H^{1}$ loops on $R^{m}$ ) by the equivalence relation " $\sim$ ": we say that $\gamma_{1} \sim \gamma_{2}$ if and only if

$$
\gamma_{1}(s)-\gamma_{1}(0)=k\left(\gamma_{2}(s)-\gamma_{2}(0)\right)
$$

for a non-zero constant $k$. The $S^{1}$-action on $\Omega R^{m}$ obviously respects this equivalence relation and projects down to a smooth action on $\Omega R^{m} / \sim$ and so for $G \times\left(\Omega R^{m} / \sim\right) \times \Omega R^{m}$. Restrict the $S^{1}$-action on $G \times\left(\Omega R^{m} / \sim\right) \times \Omega R^{m}$ to $B \Omega_{H} F$, which is what we want.

5. Application to closed geodesics. In this section we will apply the results obtained in previous sections to the study of closed geodesics.

By a closed geodesic we mean a critical point of $E$ on $\Omega M\left(x_{0}, e\right)$ for some $x_{0} \in M$. Now we fix an $x_{0} \in F$. Since the space $\Omega M\left(x_{0}, e\right)$ has a singularity at the constant loop, we cannot apply Morse theory directly to $\Omega M\left(x_{0}, e\right)$. To overcome this difficulty, we will consider either the "punctured" space $\Omega M_{\varepsilon}\left(x_{0}, e\right)=\Omega M\left(x_{0}, e\right) \cap E_{\varepsilon}^{+}$, where $E_{\varepsilon}^{+}$is the space of loops $\gamma$ with $E(\gamma) \geq \varepsilon$, or the blow-up of the horizontal loop spaces.

Proposition. For sufficiently small $\varepsilon>0$, there is no non-trivial closed geodesic $\gamma$ with $E(\gamma)<\varepsilon$. 
Proof. We will use the asymptotics of the exponential map $e_{x}(t v)$ as $t \rightarrow 0$ (cf. [20]). Let $(x, z)$ denote local coordinates in $F, v=$ $\left(v_{1}, v_{2}\right) \in T_{\left(x_{0}, z_{0}\right)} F=R^{m} \times R^{n},(y, z)=e_{x}\left(v_{1}, v_{2}\right)$. Then

$$
\left|\begin{array}{l}
y \\
z
\end{array}\right|=\left|\begin{array}{c}
x_{0} \\
z_{0}
\end{array}\right|+\left|\begin{array}{cc}
I & 0 \\
0 & A(v)
\end{array}\right||| \begin{aligned}
& v_{1} \\
& v_{2}
\end{aligned}|+| \begin{gathered}
o\left(|v|^{2}\right) \\
o\left(|v|^{4}\right)
\end{gathered} \mid
$$

where $A$ is a (matrix valued) homogeneous polynomial of degree 2 . It is proved in [20] that

$$
\operatorname{det} A(v) \neq 0 \text {. }
$$

If there is a sequence of $v_{n} \neq 0$ such that $e_{x}\left(v_{n}\right)=x=\left(y_{0}, z_{0}\right)$, and $v_{n} \rightarrow 0$ as $n \rightarrow \infty$, then there is a $v_{0}=\left(v_{1}, v_{2}\right) \neq 0$ such that $v_{1}=0, A\left(v_{0}\right) v_{2}=0$, which is in contradiction with $\operatorname{det} A\left(v_{0}\right) \neq 0$.

From now on we assume that $\varepsilon$ is chosen so that there is no nontrivial closed geodesic with energy less than $\varepsilon$.

Proposition. Suppose that $E$ is a non-degenerate Morse function on $\Omega M\left(x_{0}, e\right)$, and the Morse indices of the closed geodesics are $m_{1} \leq$ $m_{2} \leq m_{3} \leq \ldots$. Then the Morse polynomial

$$
M(t)=\sum t^{m_{k}}
$$

satisfies $M(t) \geq P(t)$ where

$$
P(t)=\sum \operatorname{dim} H^{k}\left(\Omega M\left(x_{0}, e\right)\right) t^{k} .
$$

Proof. We first apply Morse theory to $\Omega M_{\varepsilon}\left(x_{0}, e\right)$. Then we have

$$
M(t) \geq P_{\varepsilon}(t)
$$

where

$$
P_{\varepsilon}(t)=\sum\left(\operatorname{dim} H^{k}\left(\Omega M\left(x_{0}, e\right), \Omega M\left(x_{0}, e\right)-\Omega M_{\varepsilon}\left(x_{0}, e\right)\right)\right) t^{k} .
$$

On the other hand, $\Omega M\left(x_{0}, e\right)-\Omega M_{\varepsilon}\left(x_{0}, e\right)$ is contractible (cf. the remark after Proposition 4.1), so $P(t)=P_{\varepsilon}(t)$.

REMARK. One can also use the blow-up of $\Omega_{H} F$ and its $S^{1}$ equivariant homology to study the closed geodesics, as in [5].

The following lemma gives the number of connected components of $\Omega M\left(x_{0}, e\right)$. 
Proposition. The number of connected components of $\Omega M\left(x_{0}, e\right)$ is that of the elements of $\pi_{1}(F)$.

Proof. Let $\gamma_{1}$ be a loop on $F, \gamma_{1}(0)=x_{0}$. We first want to deform $\gamma_{1}$ to a horizontal loop $\gamma$ on $F$ also satisfying $\gamma(0)=x_{0}$.

Choose a subdivision $0=t_{1}<\cdots<t_{N}=1$ such that the distance (with respect to a Riemannian metric on $F$ ) of each point $\gamma(t)$ from $\gamma\left(t_{i}\right)$ is less than $\varepsilon$ for $t \in\left[t_{i}, t_{i+1}\right]$. Connect each pair $\gamma\left(t_{i}\right), \gamma\left(t_{i+1}\right)$ by one of the horizontal geodesics, say, $\gamma_{i}:\left[t_{i}, t_{i+1}\right] \rightarrow F$. Then by the estimates in [20], each $\gamma_{i}(t)$ is within a distance $M \varepsilon^{1 / 2}$ from $\gamma\left(t_{i}\right)$. If $\varepsilon$ is small enough, $\left.\gamma\right|_{\left[t_{t}, t_{t+1}\right]}$ and $\gamma_{i}$ are in the same connected component of the space of horizontal paths connecting $x\left(t_{i}\right)$ to $x\left(t_{i+1}\right)$. Now define a horizontal loop $\gamma$ by

$$
\gamma(t)=\gamma_{i}(t), \quad t \in\left[t_{i}, t_{i+1}\right]
$$

then $\gamma$ is homotopic to $\gamma_{1}$.

Now we prove that if $\gamma_{1}, \gamma_{2} \in \Omega F\left(x_{0}, x_{0}\right)$ are homotopic, then they are in the same connected component of $\Omega F\left(x_{0}, x_{0}\right)$. Let $\gamma_{\varepsilon}$ be a family of loops connecting $\gamma_{1}, \gamma_{2}$, and then choose a subdivision $0=s_{0}<\cdots<s_{m}=1$ such that

$$
d\left(\gamma_{s_{i}}, \gamma_{s_{i+1}}\right)<\varepsilon
$$

where $\varepsilon$ is a small positive number. Then for each $i, 0 \leq i \leq m$, we can choose a broken horizontal loop $\gamma_{i}^{\prime}$ homotopic to $\gamma_{i}$ such that

$$
d\left(\gamma_{i}(t), \gamma_{i}^{\prime}(t)\right)<\varepsilon
$$

We want to prove that $\gamma_{i}^{\prime}$ and $\gamma_{i+1}^{\prime}$ are in the same connected component of $\Omega F\left(x_{0}, x_{0}\right)$. In fact, if $\varepsilon$ is sufficiently small, $D\left(\gamma_{i}^{\prime}, \gamma_{i+1}^{\prime}\right)<$ $2 \varepsilon$, since $\Omega F\left(x_{0}, x_{0}\right)$ is locally path connected by Theorem 4.2 , $\gamma_{i}^{\prime}, \gamma_{i+1}^{\prime}$ are in the same connected component of $\Omega F\left(x_{0}, x_{0}\right)$. Hence $\gamma_{0}^{\prime}=\gamma_{0}, \gamma_{m}^{\prime}=\gamma_{1}$ are in the same connected component of $\Omega F\left(x_{0}, x_{0}\right)$.

REMARK. Using the regularity result of Bar [29] for geodesics, we can show that the above result remains true even if the horizontal bundle $H$ only satisfies Hörmander's condition.

As an application, consider the three-dimensional Heisenberg group $\mathrm{H}_{3}$ and let $\gamma \subset \mathrm{H}_{3}$ be a discrete subgroup with $k$ free generators such that $H_{3} / \Gamma$ is compact. It is well known that there is a left invariant contact structure on $H_{3}$ (cf. [15], [19]). Take a $\Gamma$-invariant 
Riemannian metric on $H^{3}$ and we can get the $\Gamma$-invariant CarnotCarathéodory metric on $H_{3} / \Gamma$. By Proposition 4.9 then there are at least $k$ distinct closed geodesics on $H_{3} / \Gamma$.

Appendix. The extremal fields. In this appendix we will derive the second variation of $E$ on $\Omega M\left(x_{0}, H_{0}\right)$. Since the extremals are given by Wong's equation ([18]), we need only to study the linearization of Wong's equation. We will continue to use the left-handed fiber bundle.

According to [18], Wong's equation is

$$
\nabla_{\dot{\gamma}} \dot{\gamma}=Q(t) F_{\omega}(\dot{\gamma}, \cdot)
$$

where $Q(t)=U(t) Q(0) U^{-1}(t), U(t)$ is the parallel translation along the path $\gamma(t)$.

Now suppose we have a one-parameter family of solutions $\gamma_{\varepsilon}$,

$$
\nabla_{\dot{\gamma}_{\varepsilon}} \dot{\gamma}_{\varepsilon}=Q^{\varepsilon}(t) F_{\omega}\left(\dot{\gamma}_{\varepsilon} \cdot, \cdot\right)
$$

where $Q^{\varepsilon}(t)=U_{\varepsilon}(t) Q^{\varepsilon}(0) U_{\varepsilon}^{-1}(t), U_{\varepsilon}$ is the parallel translation along the path $\gamma_{\varepsilon}$. We want to find the equation that the vector field along $\gamma, J(t)=\partial \gamma_{\varepsilon}(t) /\left.\partial \varepsilon\right|_{\varepsilon=0}$, has to satisfy. We have

$$
\left.\frac{D}{\partial \varepsilon}\right|_{\varepsilon=0} \nabla_{\dot{\gamma}_{\varepsilon}} \dot{\gamma}_{\varepsilon}=\left.\frac{D}{\partial \varepsilon}\right|_{\varepsilon=0} Q^{\varepsilon}(t) F_{\omega}\left(\dot{\gamma}_{\varepsilon}, \cdot\right) .
$$

As is well known, the left-hand side of the above is

$$
\left(\partial^{2} J / d t^{2}\right)+R(V, J) V
$$

where $V(t)=\dot{\gamma}(t)$ and $R$ is the Riemannian curvature on the base manifold $M$. Now we compute the right-hand side of (2) (we will omit $\varepsilon=0$ for simplicity).

$$
\begin{aligned}
\partial\left(Q^{\varepsilon}(t) F_{\omega}(V, \cdot)\right) / \partial \varepsilon= & \left(D Q^{\varepsilon}(t) / \partial \varepsilon\right) F_{\omega}(V, \cdot) \\
& +Q(t)\left(D_{J} F_{\omega}\right)(V, \cdot)+Q(t) F_{\omega}(\dot{J}, \cdot)
\end{aligned}
$$

and

$$
\begin{aligned}
\partial Q^{\varepsilon}(t) / \partial \varepsilon= & U^{-1}\left(\operatorname{ad}_{\int_{0}^{t} U(s) F_{\omega}(V, J) U^{-1}(s) d s}^{*} Q(0)\right) U \\
& +U(t)(d Q(0)) / d \varepsilon) U^{-1}(t)
\end{aligned}
$$

here we have used the formula in $\S 2.3$ that

$$
\partial U^{\varepsilon}(t) / \partial \varepsilon=\int_{0}^{t} U(t) F_{\omega}(V(t), J(t)) U^{-1}(t) d t
$$


modulo the term which is independent of $J$. Then we see that the linearization of Wong's equation (and the Jacobi field for the variational problem) is the 2 nd order differential-integral operator

$$
\begin{aligned}
\left(D^{2} J / d t^{2}\right) & +R(V, J) V-Q(t)\left(\nabla_{J} F_{\omega}\right)(V, \cdot)-Q(t) F_{\omega}((D J / d t),) \\
& -U(t) \operatorname{ad}_{\int_{0}^{t} U F_{\omega}(V, J) U^{-1} d s} Q(0) F_{\omega}(V, \cdot) U^{-1}(t)=0 .
\end{aligned}
$$

\section{REFERENCES}

[1] V. I. Arnold, V. V. Kozlov and A. I. Neishtadt, Dynamical Systems, III, v3 in the Encyclopedia of Mathematical Sciences Series, 1988, Springer.

[2] M. Atiyah and A. N. Pressley, Convexity and loop groups, Progr. Math., 36 (1983), 33-64.

[3] L. B. Bergery, Sur certaines fibrations d'espaces homogenes riemanniens, Compositio Math., 30 (1975), 43-61.

[4] J. M. Bismut, Large derivations and the Malliavin calculus, Progr. Math., vol. 45, Birkhäuser, Basel, 1984.

[5] R. Bott, Lecture on Morse theory, old and new, Bull. Amer. Math. Soc., 7 (1982), 331-358.

[6] R. Brockett, Control theory and singular Riemannian geometry, in New Directions in Applied Mathematics, Springer-Verlag, New York (1981).

[7] Chan Hong Mo and Tsou Sheung Tsun, Equation of motion for non-abelian monopoles, in Proc. Monopole Meeting, Trieste, Italy, ed. N. S. Craigie, P. Goddard, N. N. Nahm, 261-272.

[8] D. A. Dubrovin, A. T. Fomenko and S. P. Novikov, Modern Geometry vol. 2, (1985), Springer.

[9] Zhong Ge, Carnot-Caratheodory metrics and the horizontal paths spaces, to appear.

[10] M. Gromov, Sur Structures Métriques pour les Variétés Riemanniens, (1981), Cedic-Nathan.

[11] U. Hamenstadt, Some regularity result for Carnot-Caratheodory metrics, preprint.

[12] S. Helgason, Differential Geometry and Symmetric Spaces, (1962), Academic Press.

[13] R. Hermann, Some differential-geometric aspects of the Lagrangian variational problem, Illinois J. Math., 6 (1962), 634-673.

[14] W. Klingenberg, Riemannian Geometry, Walter de Gruyter, 1982.

[15] A. Koranyi, Geometric properties of Heisenberg-type group, Adv. Math., 56 (1985), 28-38.

[16] J. Milnor, Morse Theory, Ann. Math. Studies 51, Princeton Univ. Press, 1963.

[17] J. Mitchell, On Carnot-Caratheodory metrics, J. Differential Geom., 21 (1985), $35-45$.

[18] R. Montgomery, Shortest loops with a fixed holonomy, MSRI preprint (1988).

[19] P. Pansu, Croissance des boules et quasiisometries dans les nilvarieties, Ergodic Theory Dynamical Systems, 3 (1983), 41-445.

[20] R. S. Strichartz, Sub-Riemannian geometry, J. Differential Geom., 24 (1986), 221-263.

[21] M. Tamm, subanalytical sets in the calculus of variations, Acta. Math., 146 (1981), 167-199. 
[22] T. J. S. Taylor, Some aspects of differential geometry associated with hypoelliptic second order operators, Pacific J. Math., 136 (1989), 355-378.

[23] Tsou Sheung Tsuh, Study of monopoles using loop space, in Proceeding Monopole Meeting, Trieste, Italy, ed. N. S. Cragie, P.Goddard, N. N. Nahm.

[24] S. Webster, Pseudo-Hermitian structure on a real hyperplane, J. Differential Geom., 13 (1978), 25-40.

[25] A. Weinstein, Fat bundle and symplectic manifolds, Adv. in Math., 37 (1980), 239-250.

[26] _ Private Communication.

[27] S. Smale, Regular curves on Riemannian manifolds, Trans. Amer. Math. Soc., 87 (1958).

[28] C. B. Rayner, The exponential map for the Lagrange problem on differentiable manifolds, Phil. Trans. Roy. Soc. London, ser. A, no. 1127, v. 262, pp. 299-344.

[29] C. Bar, Geodesics for Carnot-Caratheodory metrics, preprint, 1989.

Received August 15, 1989 and in revised form March 3, 1990.

UNIVERSITY OF ARIZONA

TUCSON, AZ 85721 


\section{PACIFIC JOURNAL OF MATHEMATICS EDITORS}

\author{
V. S. VARADARAJAN \\ (Managing Editor) \\ University of California \\ Los Angeles, CA 90024-1555-05 \\ Herbert Clemens \\ University of Utah \\ Salt Lake City, UT 84112 \\ THOMAS ENRIGHT \\ University of California, San Diego \\ La Jolla, CA 92093
}

R. FINN

Stanford University

Stanford, CA 94305

HeRmann FlaschKa

University of Arizona

Tucson, AZ 85721

VAUGHAN F. R. JoNES

University of California

Berkeley, CA 94720

STEVEN KERCKHOFF

Stanford University

Stanford, CA 94305
C. C. MOORE

University of California

Berkeley, CA 94720

MaRTIN SCHARLEMANN

University of California

Santa Barbara, CA 93106

HAROLD STARK

University of California, San Diego

La Jolla, CA 92093

\section{ASSOCIATE EDITORS}
R. ARENS
E. F. BECKENBACH
B. H. NeumanN
F. WolF
K. YosHIDA

\section{SUPPORTING INSTITUTIONS}

UNIVERSITY OF ARIZONA

UNIVERSITY OF BRITISH COLUMBIA

CALIFORNIA INSTITUTE OF TECHNOLOGY

UNIVERSITY OF CALIFORNIA

MONTANA STATE UNIVERSITY

UNIVERSITY OF NEVADA, RENO

NEW MEXICO STATE UNIVERSITY

OREGON STATE UNIVERSITY
UNIVERSITY OF OREGON

UNIVERSITY OF SOUTHERN CALIFORNIA

STANFORD UNIVERSITY

UNIVERSITY OF HAWAII

UNIVERSITY OF TOKYO

UNIVERSITY OF UTAH

WASHINGTON STATE UNIVERSITY

UNIVERSITY OF WASHINGTON 


\section{Pacific Journal of Mathematics}

Vol. 149, No. $1 \quad$ May, 1991

Takao Akahori and Harunori Ameku, On the Romanov kernel and Kuranishi's $L^{2}$-estimate for $\bar{\partial}_{\mathrm{b}}$ over a ball in the strongly pseudo convex

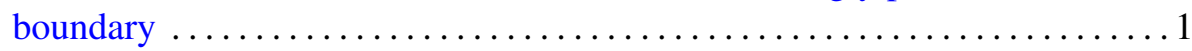

Robert Emile Beaudoin, The proper forcing axiom and stationary set

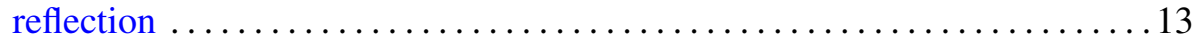

Hans Engler, A matrix Volterra integrodifferential equation occurring in

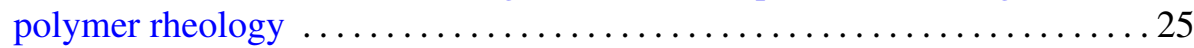

Zhong Ge, On a constrained variational problem and the spaces of

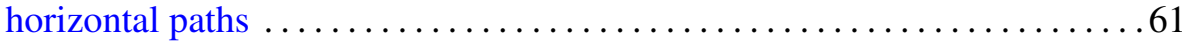

Yutaka Hemmi, Higher homotopy commutativity of $H$-spaces and the

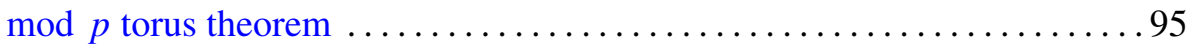

G. D. Johnson, An intrinsic characterization of a class of minimal surfaces

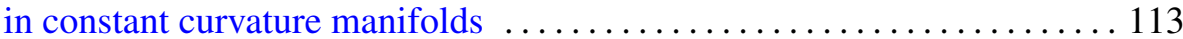

Min Ho Lee, Conjugates of equivariant holomorphic maps of symmetric domains

Jacek Nikiel, H. Murat Tuncali and Edward D. Tymchatyn, On the rim-structure of continuous images of ordered compacta

Tara Lynn Smith, Generalized Clifford-Littlewood-Eckmann groups . . . . . 157

Tara Lynn Smith, Generalized Clifford-Littlewood-Eckmann groups II:

Linear representations and applications 185 